\title{
BEITRÄGE ZUR GESCHICHTE DER 21. DYNASTIE
}

\author{
By KARL JANSEN-WINKELN
}

The main subject of the article are the relations between the rulers of Upper and Lower Egypt during the Twenty-first Dynasty. The wife of Psusennes I, whose name used to be read Mutnodjmet, is identical with Nodjmetmut, a daughter of Pajnedjem I. The supposed marriage ties between Ramses $\mathrm{XI}$ and the new royal family at Tanis cannot be substantiated and are very unlikely. An inscription at Thebes mentions a daughter of king Amenemope who was married to a High Priest of Amun, probably Pajnedjem II. It can be shown that Amenemope and Pajnedjem II were very closely allied and that they started a new political era in Upper Egypt. The history of Upper Egypt is reconsidered as well as the method of dating during this period.

\section{إضافة لتاريخ الأسرة الحادية وانسن والعثرين}

الموضوع الرئيسى لهذه المقالة هو العلاقات بين حكام مصر العليا والسفلى خلال الأسرة الحادية

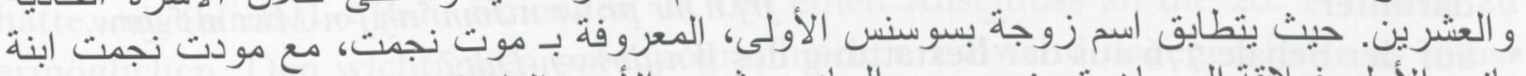

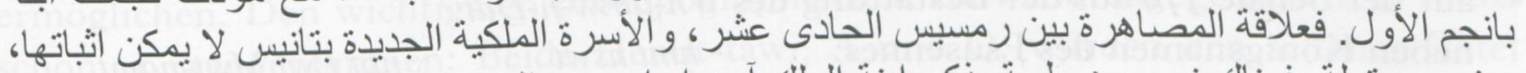

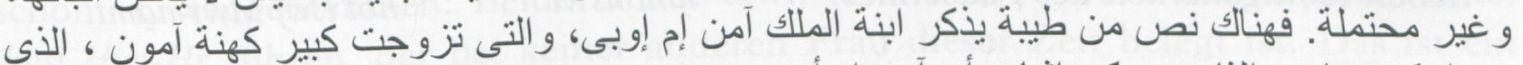

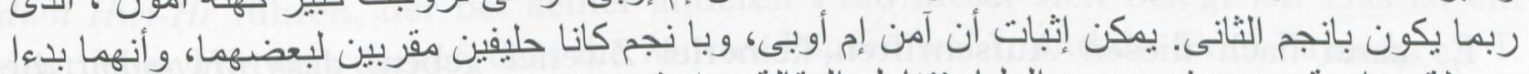
مرحلة سياسبة جديدة في مصر العليا. نتناول المقالة دراسة ناريخ مصر العليا، فضلا عن عن منهج لتأريخ هذه الفترة.

EIN charakteristisches Merkmal der 2r. Dynastie ist die Teilung des Landes in zwei Gebiete, die jeweils eigene Herrscher haben. Diese Herrscher(häuser) waren aber, wie man schon seit langem erkannt hat, keine feindlichen Rivalen, sondern offenbar freundschaftlich und meist (wenn nicht sogar immer) auch verwandtschaftlich miteinander verbunden. In dem folgenden Beitrag soll es vor allem um diese Beziehungen zwischen den Regenten von Ober- und Unterägypten gehen.

Es ist mir vollkommen klar, wieviel davon unsicher und spekulativ ist. Aber wir haben nun einmal so gut wie keine Quellen aus dieser Zeit, die konkrete Aussagen zu historischen Vorgängen machen, und sind daher weitgehend auf Schlussfolgerungen aus indirekten Hinweisen und (oft vagen) Indizien angewiesen.

\section{Mwt-ndmt oder $N \underline{d} m t-M w t$ : Die Frau Psusennes' I. und ihre thebanische Herkunft}

Der Name der Frau Psusennes' I. wird üblicherweise Mwt-ndmt (Mutnodjmet u.ä.) umschrieben. In den nicht sehr zahlreichen Belegen wird er stets in Kartusche geschrieben. Er kommt vor: 
- auf dem Sarkophag (von König Amenemope usurpiert) ${ }^{\mathrm{I}}$ und den Uschebtis ${ }^{2}$ aus ihrer ursprünglichen Bestattung im Grab des Psusennes:

Sarkophagdeckel: Wsjr hmt nswt wrt [ ... ] nbt t3wj Mwt-ndmt m3-hrw

Sarkophagwanne: Wsjr hm-ntr 2-nw n Jmn-R nswt ntrw

hmt nswt wrt tpt $n$ hm.f

wrt hnrt tpt $n$ Jmn-Re nswt ntrw

3 n pr $n$ Mwt wrt nbt Jšrw

hm-ntr $n$ Mwt wrt nbt JŠrw

hm-ntr $n$ Hnzw m Wst Nfr-htp

mwt-ntr $n$ Hnzw p3 hrd 3 wr tpj $n$ Jmn

z3t nswt

snt nswt

hmt nswt nbt t3wj Mwt-nd $m$ t $m 3^{\complement}-h r w$ hr Wsjr

Uschebtis: $\quad$ Wsjr hmt nswt Mwt-ndmt $m 3^{\top}-h r w$

- auf drei Schwalbenschwänzen im Grab des Psusennes; ${ }^{3}$ jeweils Thron- und Eigenname des Psusennes; darunter: hmt nswt snt nswt nbt t3wj Mwt-ndmt

- auf drei Beigaben der Bestattung Psusennes' I., jeweils zusätzlich zum Königsnamen: Armbänder 539/549 des Psusennes: ${ }^{4} \quad$ jrj.n ḥmt nswt wrt tpj $n$ ḥm.f nbt t3wj $M w t-n \underline{d} m t$

Schale 40 I des Psusennes: ${ }^{5}$

Schale 408 des Psusennes: ${ }^{6}$

jrj.n hrmt nswt wrt nbt t3wj Mwt-ndmt hmt nswt Mwt-ndm(t), darunter: jrj.n mr pr wr $n$ Jmn `nh.f-n-Mwt m3`-hrw

- auf der Schale 776 aus der Bestattung des $W n-\underline{d} b 3 w-n-\underline{D} d t:^{7}$ neben Königsnamen des Psusennes: hmtnswt nbt t3wj Mwt-ndmt.

Es kann nach diesen Aufschriften keinerlei Zweifel geben, dass Mwt-ndmt die Ehefrau (und Schwester) Psusennes' I. war, außerdem eine Königstochter. ${ }^{8}$ Ihr Name wird in allen Belegen in der Reihenfolge $M w t-n d m(t)$ geschrieben, und in neun von elf Schreibungen hat $n d m t$ die Endung $t$. Die Mutter Psusennes' I. war Hnwt-t3wj A, die Frau Pajnedjems I. ${ }^{9}$ Wenn also Mwt- $n d \underline{d} t$ die Schwester(gattin) des Psusennes war, sollte auch sie die Tochter Pajnedjems I. sein.

P. Montet, Les constructions et le tombeau de Psousennès à Tanis (Paris, I951), 164, fig. 60; I65-6; pl.125; Montet, Les constructions et le tombeau de Chéchanq III à Tanis (Paris, I 960), 73 (2); K. Jansen-Winkeln, Inschriften der Spätzeit, Teil I: Die 2I. Dynastie (Wiesbaden, 2007), 96 (7.5).

2 Tanis: L'or des pharaons, Ausstellungskatalog Paris/Marseille I987 (Paris, I 987), I30-I (I4); G. Martin, 'On some Shabtis of Mutnodjmet, Wife of Psusennes I', BSEG 7 (1982), 73-8; Jansen-Winkeln, Inschriften, I, 6 I (4.107).

3 Montet, Psousennès, 24, fig. 6; L'or des pharaons, I 90-I (53); Jansen-Winkeln, Inschriften, I, 47 (4.22).

$4 \quad$ Montet, Psousennès, I 50/I 53, pl. CXX; L'or des pharaons, 258-9 (92); Jansen-Winkeln, Inschriften, I, 53 (4.40); P. Le Guilloux, Le mobilier funéraire de Psousennès Ier (Paris, 2010), I 57-8 (202-3).

5 Montet, Psousennès, I02; 99, fig. 4I (rechts); pl. LXX; Jansen-Winkeln, Inschriften, I, 58 (4.83); Le Guilloux, Le mobilier funéraire, $\mathrm{I}_{3}$ ( (104).

$6 \quad$ Montet, Psousennès, I04; 98, fig. 39; pl.LXXI; Jansen-Winkeln, Inschriften, I, 59 (4.90); Le Guilloux, Le mobilier funéraire, 120 (I I I).

7 Montet, Psousennès, 84-5, fig. 32; pl. LVI; Jansen-Winkeln, Inschriften, S I, 60 (4.99).

Und nicht etwa die Mutter Psusennes' I, wie Montet angenommen hatte, s. dazu K. A. Kitchen, The Third Intermediate Period in Egypt (IIO0-650 BC) (3. Auflage) (Warminster, 1996), \$ 40 (ii).

$9 \quad$ Vgl. Kitchen, Third Intermediate Period, $\S 43$ (zu seiner Einschränkung am Ende s.u.) 
In einer (nicht zur regulären Dekoration gehörigen) Szene im Luxortempel ${ }^{10}$ ist Pajnedjem I. räuchernd und libierend vor den thebanischen Göttern dargestellt, mit den Titeln jrj-p`t hrj-tp t3wj hm-ntr tpj $n$ Jmn-R` nswt ntrw, und hinter ihm drei Frauen (bzw. ein Mädchen und zwei Frauen), die jeweils als z3t nswt $n$ ht.f mrjj. $f$ bezeichnet werden: die Gottesgemahlin Maatkare (A), die Sängerin des Amun Henuttawi (B) und eine hrj(t) $h n r w\left(\right.$ ?) $N d \underline{d} m-M w t$. Da Henuttawi B nachweislich eine Tochter Pajnedjems ist ${ }^{1 \mathrm{I}}$ und dies auch für Maatkare A mit guten Gründen angenommen wird, ${ }^{12}$ wird hier zweifellos Pajnedjem mit drei seiner Töchter dargestellt. Wenn also diese $N \underline{d} m-M w t$ eine Tochter Pajnedjems I. ist ${ }^{\mathrm{I} 3}$ und die Königin $M w t-n \underline{d} m t$ ebenso, stellt sich die Frage, ob diese beiden nicht identisch sind, denn es ist doch sehr unwahrscheinlich, dass Pajnedjem eine Tochter namens $M w t-n \underline{d} m t$ und eine zweite mit Namen $N \underline{d} m-M w t$ hatte.

Sofern bei den Belegen aus Tanis der Gottesname vorangestellt wurde, könnte man ja dort ohne weiteres $N \underline{d} m(t)$ - $M w t$ lesen. Allerdings müsste man dann, wegen des häufigen $t$, den Namen nicht als $N \underline{d} m-M w t$ ("Mut ist angenehm"), sondern als $N \underline{d} m t-M w t$ ("Die Angenehme der Mut”) ${ }^{14}$ verstehen. Unterschiedliche Schreibungen eines solchen Namens in Tanis und Theben wären kaum verwunderlich, da ja sicher unterschiedliche Schreiber dafür verantwortlich waren.

Allerdings hatte Kitchen bestritten, ${ }^{15}$ dass die in Luxor dargestellte $N \underline{d} m(t)-M w t$ mit der Mwt-ndmt aus Tanis identisch sei. Dass er von zwei unterschiedlichen Frauen ausging, liegt daran, dass er Psusennes I. und Mwt-nd $\underline{m} t$ nicht als Nachkommen von Pajnedjem I. bzw. Henuttawi A ansetzte, sondern eine Henuttawi Q erschaffen hatte, angeblich die Frau Smendes' I., um einen Anschluss an die 20. Dynastie zu ermöglichen. Den wichtigsten prosopographischen Einwand dagegen hatte er selbst schon vorweggenommen: Beide Henut-tawi, A und $\mathrm{Q}$, würden den seltenen Titel $d w 3 t H w t-H r$ führen, der bei keiner anderen Frau dieser Zeit belegt ist. Das ist ein deutlicher Hinweis darauf, dass die Mutter Psusennes' I. und die Frau Pajnedjems I. ein und dieselbe Person ist. Kitchen hat in der zweiten Auflage seines Buches diese Theorie selbst revidiert und geht nun stattdessen von einer doppelten T3-nt-Jmn (A und B) aus. ${ }^{16}$ Das heißt, in dieser neueren Rekonstruktion des Familienstammbaums gibt es nur noch eine einzige Henuttawi als Frau Pajnedjems I. und Mutter Psusennes' I. Da sie kaum zwei Töchter mit den Namen $M w t-n \underline{d} m t$ und $N \underline{d} m(t)-M w t$ gehabt haben dürfte, wird es sich bei der in Luxor dargestellten $N \underline{d} m(t)-M w t$ und der in Tanis dokumentierten Frau Psusennes' I. mit großer Wahrscheinlichkeit um ein und dieselbe Person handeln. ${ }^{17}$

G. Daressy, 'Notes et remarques', RecTrav I4 (1893), 32 (LII); E. Wente, 'On the Chronology of the Twenty-first Dynasty', FNES 26 (1967), r67-8; M. Römer, Gottes- und Priesterherrschaft in Ägypten am Ende des Neuen Reiches (ÄAT 21; Wiesbaden, I 994), 55I-2 (I9); Reliefs and Inscriptions at Luxor Temple, II (OIP I I6; Chicago, 1998), 52-3; pl. I99/200; Jansen-Winkeln, Inschriften, I, I 7 (22.a).

II Vgl. Jansen-Winkeln, Inschriften, I, I9I-2 (I I.20)

12 Vgl. Kitchen, Third Intermediate Period, $\$ 48$.

13 So auch A. Dodson, Afterglow of Empire (Kairo, 2012), 45.

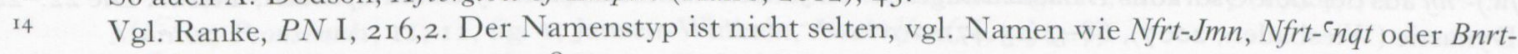
Mwt etc., s. Ranke, PN I, 201, I 5; 20; 97, I 8 .

${ }_{5} \quad$ Kitchen, Third Intermediate Period, $\$ 40$ (I).

I6 Kitchen, Third Intermediate Period, § $44 \mathrm{I}-3$.

17 Ebenso Dodson, Afterglow of Empire, 46; 60. In Kitchens neuen Stammbäumen (Third Intermediate Period, § 44I) wird die $N \underline{d} m(t)$-Mwt aus Luxor nicht mehr erwähnt. 


\section{Die angebliche ramessidische Verwandtschaft der 21. Dynastie}

Die Annahme einer Heiratsverbindung der Königshäuser der 20. und 21. Dynastie ist sehr verbreitet und wird in nahezu allen neueren Arbeiten vertreten. ${ }^{18}$ Es gibt dafür einen spezifischen und einen sehr allgemeinen Grund: Der spezifische, der vor allem von Kitchen angeführt und ausgearbeitet worden ist, besteht darin, dass Psusennes in zwei Belegen die Namenskombination Ramses-Psusennes in der Kartusche führt und dass sich sein (angeblicher) Sohn Anchefenmut C ebenfalls zweimal RamsesAnchefenmut nenne. Der allgemeine Grund, für die meisten vermutlich noch wichtiger, ist die Annahme, die neue Dynastie habe sich auf diese Weise Legitimation verschaffen wollen (und müssen). Keine der beiden Begründungen ist überzeugend.

Der "Ramses"-Titel des `nh.f-n-Mwt C ist dreimal als $z 3 n s w t R^{\ulcorner}-m s j-s w$ bzw. $z$ 3 nswt n $\underline{h t}$.f mrjj.f $R^{\complement}-m s j-s w$ auf Gegenständen aus seiner Bestattung belegt:

- Westwand des Grabes, Z.ı 8:19 $\quad[\ldots] z 3 n s w t R^{\top}-m s j-s w[\ldots]$

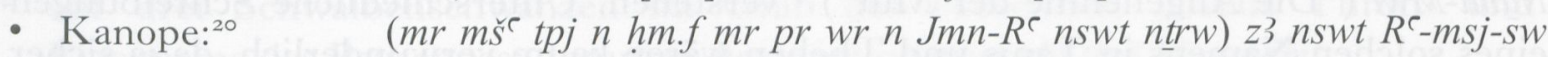
'nh.f-n-Mwt

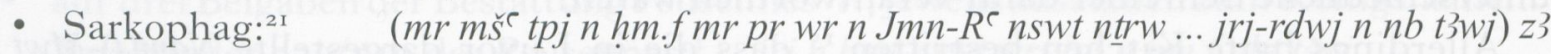
$n s w t n \underline{h t}$.f mrjj.f $R^{\top}-m s j-s w ' n h . f-n-M w t$.

In der ersten Form ( $\left.z 3 n s w t R^{\complement}-m s j-s w\right)$ entspricht er dem in der 22. Dynastie nicht seltenen "Titel" $z 3$ nswt (n) $R^{\top}-m s j-s w$, der zuletzt von Ph. Collombert ${ }^{22}$ und F. Payraudeau ${ }^{23}$ besprochen worden ist. Der jüngste Vorschlag von Payraudeau, dass er eine bestimmte (verwandtschaftliche) Beziehung zum König ausdrückt, dürfte gegenüber den früheren Deutungen des Titels den Vorzug verdienen, auch wenn die genaue Art dieser Beziehung für uns undeutlich bleibt. ${ }^{24}$

In der zweiten Form, mit $n$ ht.f mrjj.f zwischen $z 3$ nswt und $R^{\top}-m s j-s w$, ist der Titel “Königssohn des Ramses” allerdings sonst (in der 22. Dynastie) nie belegt, und deshalb hat Kitchen energisch bestritten, `nh.f-n-Mwt könne überhaupt solch ein "Königssohn des

I8 Z.B. Kitchen, Third Intermediate Period, § 4I; 220; § 439-43; A. Niwiński, 'Problems in the Chronology and Genealogy of the XXIst Dynasty: New Proposals for Their Interpretation', JARCE I6 (I979), 50-I; N. Grimal, Histoire d l'Egypte ancienne (Paris, 1988), 377; J. Taylor, 'The Third Intermediate Period (I069-664 BC)', in I. Shaw (ed.), The Oxford History of Ancient Egypt (Oxford, 2000), 33 I; D. O'Connor, 'New Kingdom and Third Intermediate Period, I 552-664 BC', in B. G. Trigger, B. J. Kemp, D. O'Connor, A. B. Lloyd (eds), Ancient Egypt: A Social History (Cambridge, I983), 234; A. Dodson and D. Hilton, The Complete Royal Families of Ancient Egypt (London, 2004), 187/200; D. Aston, Burial Assemblages of Dynasty 2I-25. Chronology - Typology Developments (CCEM 21, Wien 2009), 4I9 (22) und sonst oft. Im Bonner Totenbucharchiv wird für den Papyrus Kairo JE 95887 (= pBoulaq 23) der Hnwt-t3wj A unter "Verwandte" der nirgends belegte Ramses XI. als Vater angeführt, nicht dagegen die tatsächlich bezeugte Mutter T3-nt-Jmn. Das zeigt, wie tief verwurzelt diese Irrlehre ist.

19 Montet, Psousennès, 67; pl.XXXVIII (oben); Jansen-Winkeln, Inschriften, I, 62 (4. I 09).

20 Montet, Psousennès, 58 (308); 59, fig.2 I; Jansen-Winkeln, Inschriften, I, 63 (4. I I 2 ).

${ }_{21}$ Montet, Psousennès, 65-6; pl.XXXIX; Jansen-Winkeln, Inschriften, I, 62-3 (4. I I I).

22 Ph. Collombert, 'Les "fils Royaux de Ramsès": Une nouvelle hypothèse', GM I 5I (1 996), 23-35.

23 F. Payraudeau, Administration, société et pouvoir à Thèbes sous la XXIIe dynastie bubastite (BdE $\mathrm{r} 60$; Kairo, 2014), 328-9. In beiden Aufzählungen der Belege wäre die Bronzestatuette der Göttin Mut des $\underline{D} d-B 3 s t t$ jw.f- nh aus der Zeit Osorkons I. nachzutragen, s. K. Jansen-Winkeln, Inschriften der Spätzeit, Teil II: Die 22.-24. Dynastie (Wiesbaden, 2007), 68-9 (13.47).

24 Im Übrigen ist es auffallend, dass eine Person unter den zwölf (oder I3, vgl. Jansen-Winkeln, Inschriften, II, 393-4 [44.26]) Personen mit diesem Titel nach Namen und Determinativ (gegen D. Meeks, 'Les donations aux temples dans l'Egypte du Ier Millénaire avant J.-C.', in E. Lipiński [ed.], State and Temple Economy in the Ancient Near East, II [OLA 6; Leuven, I979], 631, n.102) zweifellos eine Frau ist, vgl. Jansen-Winkeln, Inschriften, II, 60 (13.36). 
Ramses" sein; ${ }^{25}$ die Verbindung sei vielmehr in "(leiblicher) Königssohn” und "RamsesAnchefenmut" zu trennen. Das ist aber keineswegs sicher, da der Titel hier zum ersten Mal bezeugt wäre und in seiner Form noch variabel sein könnte. Als "indirekter Genetiv" (n) $R^{\complement}-m s j-s w$ verstanden, wäre die Verbindung grammatisch unproblematisch, und eine (ältere) Variante “leiblicher Königssohn des Ramses” wäre a priori keineswegs undenkbar.

Gleichgültig aber, ob diese Titulatur als "(leiblicher) Königssohn des Ramses Anchefen-mut” oder als “(leiblicher) Königssohn Ramses-Anchefenmut” zu verstehen ist, in beiden Fällen dürfte sie keine verwandtschaftliche Beziehung zu den Ramessiden bezeichnen, da Anchefenmut zwar ein Mitglied der Königsfamilie war, aber sehr wahrscheinlich nicht der Sohn des Psusennes, sondern des Herihor ${ }^{26}$ oder Pianch, ${ }^{27}$ für die noch nie jemand ramessidische Verbindungen vermutet hat.

Die Variante Ramses-Psusennes findet sich nur zweimal unter weit mehr als hundert Belegen für den Königsnamen des Psusennes:

- auf einem in einem Ofen gefundenen Kalksteinfragment, in Kartusche: ${ }^{28}$

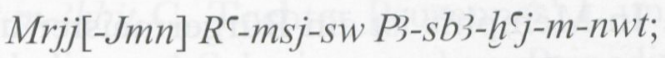

- auf einem Ring (7I4) aus der Bestattung des Wn- $\underline{d} b 3 w-n-\underline{D} d t$, in Kartusche: ${ }^{29}$ $<$ Mrjj>-Jmn Re-msj-sw P3-sb3-h-j-m-nwt.

Aus diesem sporadischen Zusatz, der sonst bei keinem anderen König der 2 I . Dynastie mehr vorkommt, ${ }^{3 \circ}$ wird man schwerlich schließen können, dass Ramses XI. der Großvater des Psusennes war. Eine Abstammungsangabe in einer Kartusche wäre mehr als unüblich. Dagegen erscheint der Titel ḥm-ntr tpj $n$ Jmn bei Psusennes (und Amenemope) mehrfach in der Kartusche mit dem Eigennamen. Es läge also näher, dass auch "Ramses" eine Art Titel ist; auch die Verbindung "Königssohn des Ramses" wird ja wie ein Titel gebraucht und eben nicht wie eine genealogische Angabe. ${ }^{3 \text { I } ~ " R a m s e s " ~}$ dürfte daher in diesen Gebrauchsweisen eher eine zusätzliche Königsbezeichnung sein, vergleichbar dem Namen "Caesar", der bald zu einer Rangbezeichnung wird (die allerdings von ungleich längerer Dauer war).

Falls der Zusatz hingegen als Hinweis auf (reale oder fiktive) Verwandtschaft mit den Ramessiden der Legitimation der eigenen Herrschaft dienen sollte, warum ist er dann so selten? Und warum erscheint er nicht in den ausführlichen Titulaturen und auf den Sphingen und anderer Großplastik, die im Tempel öffentlich aufgestellt war? Wenn die Verbindung Ramses-Psusennes auf eine Verwandtschaft mit den Ramessiden anspielte, hätte Psusennes sie seiner Mutter Henuttawi zu verdanken. ${ }^{32}$ Gerade

25 Kitchen, Third Intermediate Period, § 4I, n.220; ebenso Collombert, GM I 5I, 29. Der ramessidische Prinz (Re-msj-sw-)Mntw-hr-hpšf kann, gegen Collombert und Kitchen, nicht als Vorbild oder Parallele zu $\left(R^{\top}-m s j-s w-\right)^{\top} n h . f-n-M w t$ dienen: in solchen ramessidischen Prinzennamen gehört Ramses zum Namen, denn das Suffix bezieht sich darauf. Die Verbindung Ramses + theophorer Name kommt daher nur in diesem Namenstyp

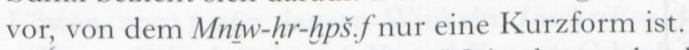

26 Jansen-Winkeln, 'Der Majordomus des Amun Anchefenmut', $D E_{3} 8$ (1997), 29-35.

27 U. Rössler-Köhler, 'Pianch - Nedjmet - Anchefenmut - eine Kleinigkeit', GM I67 (I 998), 7-8.

28 Montet, Psousennès, I I ; I2, fig. I; Jansen-Winkeln, Inschriften, I, 44 (4.7).

29 Montet, Psousennès, 75; 74, fig. 27, links; pl. LIII; Jansen-Winkeln, Inschriften, I, 60 (4.98).

30 Es ist allerdings zu berücksichtigen, dass wir für Smendes nur sehr wenige Belege haben und nicht ca. I 20 wie für Psusennes.

${ }^{31} \quad$ Zumindest für die 22. Dynastie ist das unstrittig.

32 Kitchen, Third Intermediate Period, \$ $44 \mathrm{I}$. 
Henuttawi macht sehr viele Angaben zu ihren verwandtschaftlichen Beziehungen, ${ }^{33}$ aber eine Abstammung von Ramses XI. ist nicht darunter, und die hätte man gewiss nicht verschwiegen, wenn sie der Legitimation der Dynastie dienen sollte.

Es ist zudem aus allgemeinen Erwägungen heraus ganz unwahrscheinlich, dass die 2I. Dynastie einen wie auch immer gearteten Anschluss an die Ramessiden gesucht hätte, da mit ihr etwas ganz Neues beginnt und in vielerlei Hinsicht mit alten Traditionen gebrochen wird, gerade was den königlichen Bereich angeht: Residenz und Königsnekropole werden verlegt, in Königsgräbern und königlichem Totenkult gibt es keinerlei Kontinuität, die Namen folgen anderen Mustern etc. Unter den ersten Königen der 2 I. Dynastie ist die Deltaresidenz von der Ramsesstadt nach Tanis verlagert worden. Hätte man Wert darauf gelegt, sich in die 'Tradition der Ramessiden zu stellen, hätte man ja den Namen "Haus des Ramses” beibehalten können, aber das hat man nicht getan. Es ist auch zweifellos bezeichnend, dass sich in der gesamten 2 I . Dynastie kein einziger Nachkomme der ramessidischen Königsfamilie mehr findet, die Tradition ist radikal abgeschnitten worden. Eine Tradition früherer Könige ist in der 2I. Dynastie generell kaum nachzuweisen. In Memphis ist ein Bürgermeister u.a. Prophet des "Amun des Sethos" (hm-ntr ... n Jmn n Stj), ${ }^{34}$ und ein Prophet des Königs Sahure aus dem Alten Reich ist gleichfalls belegt, ${ }^{35}$ aber kein Ramses.

Es lässt sich nicht der geringste Anhaltspunkt dafür finden, weder in den Quellen noch in der historischen Wahrscheinlichkeit, dass die Herrscherfamilie der 21. Dynastie mit der Königsfamilie der 20. Dynastie verwandt oder verschwägert war (oder es auch nur sein wollte). Alle derartigen Versuche sind willkürliche und ganz unwahrscheinliche Konstruktionen.

\section{Eine Tochter des Amenemope in Theben}

Im Jahr I 995 hat A. Cabrol eine kurze Inschrift veröffentlicht, ${ }^{36}$ deren Bedeutung für die Geschichte der 2 I. Dynastie bis jetzt noch nicht erkannt oder jedenfalls noch nicht erörtert worden zu sein scheint. Auf der Vorderseite (der Statuette) des Sphinx Nr.2o aus der Sphingenallee vor dem Muttempel ist noch der Beginn von zwei Kolumnen erhalten:

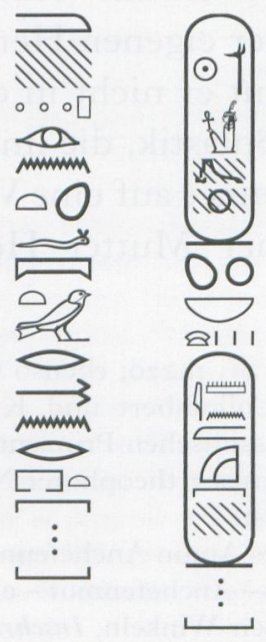

33 Jansen-Winkeln, Inschriften, I, 84-7 (6.28).

34 K. Jansen-Winkeln, 'Zu zwei Personen der frühen Dritten Zwischenzeit', SAK 35 (2006), 135-6; Jansen-Winkeln, Inschriften, I, I 56 (9.54).

35 Jansen-Winkeln, Inschriften, I, I I4 (9.13).

${ }_{3} 6$ A. Cabrol, 'Une représentation de la tombe de Khâbekhenet et les dromos de Karnak-Sud: nouvelles 
Das ist schon insofern sehr bemerkenswert, als die Könige der 2. Hälfte der $2 \mathbf{I}$. Dynastie in Theben sonst keinerlei Spuren monumentaler Aktivitäten hinterlassen haben, weder Amenemope noch Siamun oder Psusennes II. Und auch diese kleine (Usurpations-)Inschrift geht nicht eigentlich auf den König Amenemope zurück, sondern auf eine Tochter von ihm. Jedenfalls ist dies die naheliegende, wenn nicht offensichtliche Interpretation, denn das Suffix von $z 3 t . f$ kann sich kaum auf Amun beziehen. Eine Gottesgemahlin kann sich als "Tochter" des Amun bezeichnen, für die hrjt wrt hnrt (tpt) ist das nie belegt und auch nicht anzunehmen, selbst für die Hohenpriester ist es nicht üblich, sofern sie nicht gleichzeitig Könige sind. Auf Sphinx Nr. I4 steht eine entsprechende Inschrift von einer Tochter Pajnedjems I., ${ }^{37}$ die gleichfalls den Titel einer hrjt wrt [hnrt (tpt)] führte, also vermutlich auch die Frau eines Hohenpriesters war. Es wäre also möglich, dass sich die Ehefrauen der Hohenpriester hier vor dem Mut-Tempel als Stifterinnen verewigen durften.

Bei Nr. I4 könnte es sich um eine bislang nicht identifizierte Tochter Pajnedjems handeln, die zugleich die Frau des Masaharta war, oder möglicherweise um 3st-m-3hbjt C, Tochter Psusennes' I. und Ehefrau des Mencheperre, die also zugleich Enkelin und Schwiegertochter Pajnedjems I. war. Man hätte sie daher zweifellos als "seine Tochter" bezeichnen können.

Bei Nr.20 besteht kein Zweifel, dass es sich um eine Tochter des tanitischen Königs Amenemope handelt, die bisher nicht bekannt ist, wie überhaupt die Verwand tschaftsverhältnisse des Amenemope ganz unbekannt sind. ${ }^{38}$ Ihrem Titel nach müsste sie die Frau eines Hohepriesters gewesen sein, und eine derart hohe Stellung ist für eine Königstochter ja auch zu erwarten. Angesichts der Tatsache, dass wir so wenig über die tanitischen Könige der 2r. Dynastie wissen, ist diese neuerliche Verbindung zum oberägyptischen Herrscherhaus sicherlich bemerkenswert.

Da die Prinzessin eine hrjt wrt hnrt $[(t p t) n J m n]$ war, fragt es sich zunächst, mit welchem Hohenpriester sie verheiratet war. Mencheperre, der vermutlich kurz nach Regierungsbeginn des Amenemope in höherem Alter starb, kommt nicht in Frage, und Psusennes III. war noch nicht Hohepriester. Die beiden einzigen Kandidaten sind Smendes II. und Pajnedjem II. Für beide sind zwei Ehefrauen bekannt. Smendes II. war (sehr wahrscheinlich) mit Hnwt-t3wj $\mathrm{C}^{39}$ verheiratet sowie mit T3-hntDhwtj, die in den Dekreten für Ns-Hnzw A als deren Mutter genannt wird. ${ }^{4 \circ} H$ Hnwtt3wj $\mathrm{C}$ ist die Tochter von 3st-m-3hbjt $\mathrm{C}$ und Mencheperre und kommt damit nicht als die gesuchte Prinzessin in Betracht. Die Eltern von T3-hnt-Dhhwtj sind nicht bekannt, sie käme also in Frage. Eine weitere Ehefrau für diesen nur sehr kurz amtierenden Regenten wäre natürlich möglich, ist aber nicht unbedingt wahrscheinlich. Überhaupt spricht einiges dafür, dass die nur sehr kurze Amtszeit des Smendes, die möglicherweise

hypothèses', Cahiers de Karnak X (1995), 45-6; vgl. auch A. Cabrol, Les voies processionnelles de Thèbes (OLA 97; Löwen, 2001), 262; Jansen-Winkeln, Inschriften, I, I00 (7.31).

37 Cabrol, Cahiers de Karnak X, 45-6; Cabrol, Les voies processionnelles, 262; Jansen-Winkeln, Inschriften, I, I6 (3.21). Die Tatsache, dass sich einige Schmuckstücke Psusennes' I. in der Bestattung des Amenemope befanden (Montet, Psousennès, г67-8, pl. CXXXIV (Nr.643); г 7ı, pl. CXXXVII (Nr.653/654); I 72, I 70, fig. 63 (Nr.668); Jansen-Winkeln, Inschriften, I, 59-60 [4.94-96]), könnte allerdings ein Hinweis darauf sein, dass Psusennes der Vater des Amenemope war.

39 Kitchen, Third Intermediate Period, $\$ 46$ (II); 47; J. Winand, 'Les décrets oraculaires pris en l'honneur d'Henouttaouy et de Maâtkarê (Xe et VIIe Pylônes)', Cahiers de Karnak XI (2003), 630-r; 632-3.

40 Jansen-Winkeln, Inschriften, I, I I9-2I (9.27); I 22-4I (9.3 I; 9.32). 
mit der letzten Phase des Mencheperre zusammenfiel, sehr turbulent war (s.u.), und daher nicht der geeignete Moment für Ausschmückungen des Muttempels.

Ein sehr viel näherliegender Kandidat als Ehemann der Tochter des Amenmope ist Pajnedjem II., mit dessen Amtszeit nahezu die gesamte Regierung des Amenemope parallel läuft. Die bekannten Frauen Pajnedjems sind 3st-m-3hbjt $\mathrm{D}^{41}$ und Ns-Hnzw A. ${ }^{42}$ Wenn ihre Väter, wie allgemein angenommen, Mencheperre bzw. Smendes II. sind, kommen beide nicht als Töchter des Königs Amenemope in Betracht. Zumindest bei Ns-Hnzw ist dies allerdings nicht wirklich sicher; die Identität der Frau Pajnedjems II. mit der im Orakeldekret der Hnwt-t3wj genannten Ns-Hnzw, der Tochter Smendes II., ist nur eine weitgehend akzeptierte Annahme, die durchaus auch in Frage gestellt werden könnte, da die $N s-H n z w$ des Orakeldekrets ihr Vermögen ihrer Tante vermacht, während $N s$ - Hnzw A doch selbst vier Kinder hatte. ${ }^{43}$ Auch die Tatsache, dass gerade Ns-Hnzw A als “Königssohn von Kusch” einen für eine Frau extrem ungewöhnlichen Titel führt, könnte vielleicht auf eine ungewöhnlich hohe Abstammung schließen lassen. Aber es gibt etwas, das deutlich dagegen spricht, dass sie die Tochter des Amenemope war: In all den (für die Zeit) verhältnismäßig zahlreichen Zeugnissen unterschiedlicher Art, in denen ihr Name und ihre Titel erwähnt werden, wird sie niemals "Königstochter" genannt. Tatsächlich wird ihr Vater nie direkt genannt, ${ }^{44}$ und auch die Mutter nur in den Totendekreten. ${ }^{45}$

Vielleicht sollte man sich daher nach einer anderen Kandidatin umsehen. ${ }^{46}$ Es gibt eine weitere hrjt wrt hnrt tpt $n$ Jmn namens $\underline{D} d-M w t-j w . s-{ }^{\complement} n h$, die nur von ihrer Bestattung im "Grab der Prinzessinnen" in Deir el-Bahari her bekannt ist. ${ }^{47}$ Ihre Särge werden in die späte 2 I. Dynastie, in die Zeit der HPA Pajnedjem II. oder Psusennes III. datiert, ${ }^{48}$ so dass sie zeitlich in Frage käme. Auch sie wird nicht als $z 3 t$ nswt bezeichnet, und die Inschriften auf ihrer Grabausstattung nennen weder Mutter noch Vater. Aber an ihrem Kopf war ein Uräus befestigt, ${ }^{49}$ und daraus sollte man auf königliche Abkunft schließen..$^{50}$ Die teilweise recht ungewöhnlichen Titel der $D d-M w t-j w . s-{ }^{\top} n h$ geben keinen Aufschluss über ihre Herkunft.

$\mathrm{Ob}$ aber nun sie oder $\mathrm{Ns}$ - Hnzw A die gesuchte Tochter des Amenemope war, oder eine möglicherweise noch unbekannte Dritte, die Tatsache selbst, dass Amenemope eine Tochter nach Theben gab, ist sehr bemerkenswert, denn es gibt Anzeichen dafür, dass sich mit dem

\footnotetext{
${ }^{\mathrm{I}} \quad$ Kitchen, Third Intermediate Period, $\$ 52$ (D).

42 Kitchen, Third Intermediate Period, $\$ 53$ (A). Die Zweifel von Winand, Cahiers de Karnak XI, 2003, 632, dass Ns-Hnzw die Ehefrau von Pajnedjem II. war, sind kaum gerechtfertigt.

43 Vgl. Winand, Cahiers de Karnak XI (2003), 669.

44 Auch im Dekret der Hnwt-t3wj C ist Smendes II. als Vater der Ns-Hnzw nur zu erschließen.

45 Sollte diese Mutter T3-hnt-Dhwtj mit der in der 2. Cachette bestatteten T3-hnt-Dhwtj / T3-hnt-nb-Hmnw (Bestattung A.4I) identisch sein, wie das L. Aubert, Les statuettes funéraires de la Deuxième Cachette à Deir el-Bahari (Paris, I998), 93 erwogen hat, wäre das ein deutlicher Hinweis darauf, dass sie nicht die Frau des Königs Amenemope war.

$4^{6}$ Dass Pajnedjem II. mehrere Ehefrauen hatte, wird im Totendekret der Ns-Hnzw A expressis verbis erwähnt, s. Jansen-Winkeln, Inschriften, I, I 26 (9.3 I, Z.25); I37 (9.32, Z.82).

47 Grab Nr. 6o, neben dem Vorhof des Tempels der Hatschepsut, s. PM I ${ }^{2}, 629$; H. Winlock, BMMA, 'The Egyptian Expedition 1923-I 924, II, December 1924', 21-33, bes. 26-8, fig. 28-9; 'I924-1925, March I926', 1 8-32, bes. 1 8-19, fig. 17-1 8; 24-5, fig. 26-7; 27, fig. 32; Aston, Burial Assemblages, 199-200 (TG 830); JansenWinkeln, Inschriften, I, I 92-3 ( I I.2 I); S.-A. Naguib, Le clergé feminin d'Amon thébain à la 2re Dynastie (OLA 38 ; Löwen, I990), I73-5.

48 A. Niwiński, 2 Ist Dynasty Coffins from Thebes: Chronological and Typological Studies (Theben 5; Mainz, 1988), I60 (Nr. 308); Niwiński, ҰARCE I6, 54.

49 Winlock, BMMA, 'The Egyptian Expedition I924-1925, II', 24.

$5^{\circ}$ Vgl. Kitchen, Third Intermediate Period $\$ 54$ (C), der sich aber auf thebanische Kandidaten beschränkt.
} 
Beginn seiner Regierung die Verhältnisse in Theben grundlegend änderten. Und es wäre durchaus möglich, dass Amenemope und Pajnedjem II. dabei zusammengewirkt haben.

\section{Iv. König Amenemope in Theben}

Von König Amenemope ist nur recht wenig bekannt. In Tanis hat er offenbar am Tempel des (Gottes) Jmn-m-jpt arbeiten lassen ${ }^{51}$ und eine kleine Horusstatuette ${ }^{52}$ von ihm ist im Grab Osorkons II. im Sarkophag des Hornacht gefunden worden, ein weiteres Zeugnis für die enge Verbundenheit von 21. und 22. Dynastie. Die meisten Zeugnisse kommen natürlich aus seinem ungestörten Begräbnis in NRT III. ${ }^{53}$ Aus Memphis gibt es einen Block aus Mitrahina mit einer Darstellung der Sachmet ${ }^{54}$ sowie einige Fragmente aus der Isiskapelle von Giza, die schon sein Vorgänger Psusennes I. hatte ausschmücken lassen. ${ }^{55}$ In Theben stammen die einzigen Zeugnisse für Amenemope-abgesehen eben von der Inschrift seiner Tochter auf der Sphinxstatuette-aus einer Reihe von Bestattungen: Ein auf sein Jahr 5 datiertes (!) Totenbuch, ${ }^{56}$ Mumienriemen aus Deir el-Medina und der Königscachette(?) und nicht weniger als I3 Belege auf Mumienhüllen der 2. Cachette (s.u.).

Um das Besondere der thebanischen Belege für Amenemope richtig zu verstehen, muss man einen Blick auf die Beschriftungsmöglichkeiten der Bestattungen dieser Zeit insgesamt werfen. Die verschiedenen Mumienhüllen der 2 I. Dynastie können auch eine Reihe unterschiedlicher Aufschriften haben, in hieroglyphischer und hieratischer Schrift: ${ }^{57}$ a) Äußere Hüllen und Bänder (wie auch Särge): Hieratische Wiederbestattungsvermerke (praktisch nur auf Mumien der Königscachette). ${ }^{5^{8}}$

b) Grobe Leichentücher mit Osirisfigur: Titel und Namen des Toten hieroglyphisch, z.T. durch funeräre Formeln erweitert. ${ }^{59}$

c) Feine Leichentücher ("serviettes”) mit hieroglyphischem Vermerk der ausgebenden Institution: $p r-J m n$ oder $\mathrm{pr}$-Hnzw. ${ }^{60}$

d) Mumienriemen (Lederbänder) und -anhänger ("pendeloques”) mit Namen des Hohepriesters und/oder Königs, teils mit jrj.n davor.

e) Leichenbänder mit (oft datierten) hieroglyphischen Vermerken der Form mnht jrj.n HPA. $^{61}$

${ }_{51} \quad$ Jansen-Winkeln, Inschriften, I, 95 (7.1).

$5^{2} \quad$ Kairo JE 87142, P. Montet, Les constructions et le tombeau d'Osorkon II à Tanis (Paris, 1947), 66-7; Jansen-Winkeln, Inschriften, I, 95 (7.2).

53 Montet, Psousennès, I 59-72; pls CXXV-CXXXVII; Jansen-Winkeln, Inschriften, I, 95-9 (7.3-22).

${ }_{54} \quad$ Kairo TN $3 / 7 / 24 /$ I I, s. Gauthier, 'A travers la Basse-Égypte', $A S A E 22$ (1922), 204-5; C. Zivie-Coche, Giza au premier millénaire (Boston, I99I), 59; Jansen-Winkeln, Inschriften, I, 99 (7.23).

55 Zivie-Coche, Giza au premier millénaire, 59-78; Jansen-Winkeln, Inschriften, I, 99-1 00 (7.24-30).

${ }_{5} 6$ I. Munro, Das Totenbuch des Pa-en-nesti-taui aus der Regierungszeit des Amenemope (HAT 7; Wiesbaden, 2001), Taf. 68-9; Jansen-Winkeln, Inschriften, I, ro9 (7.50).

57 S. den Überblick bei K. Jansen-Winkeln, Text und Sprache in der 3. Zwischenzeit (ÄAT 26; Wiesbaden, I 994), 433-4.

$5^{8} \quad$ Sehr selten kommen auf äußeren Bändern auch Tb-Sprüche vor, vgl. etwa G. Daressy, 'Procès-verbal d'ouverture de la momie No 29707', $A S A E_{3}$ (1902), I 53; I 55-6.

59 Z.B. S. A. Abdalla, 'A Group of Osiris-Cloths of the Twenty-First Dynasty in the Cairo Museum', $\mathscr{F E A} 74$ (1988), I 57-64; pls XXI-XXIII.

6o Eine Abbildung bei H. Winlock, The Tomb of Queen Meryet-Amūn at Thebes (PMMA 6; New York, I932), pl. XLI.

6r Vgl. die Übersicht über die verschiedenen Formen bei Kitchen, Third Intermediate Period, §372-8. Zu Abbildungen dieser Leichenbänder s. Winlock, The Tomb of Queen Meryet-Amūn, pl. XL. Mit Privatpersonen kommt diese Form der Beschriftung auch vor, aber auf Leichentüchern, s. G. Daressy, 'Les cercueils des prêtres d'Ammon', $A S A E 8$ (1907), 27 (A.64); 35 (A.133). 
f) Leichenbänder und feine Leichentücher mit hieroglyphischem Vermerk jnw jrj.n NN (z.T. mit Datum). ${ }^{62}$

Für das Problem, um das es hier geht, sind nur die Gruppen d) und e) von Bedeutung. Form und Funktion der Mumienriemen und -anhänger sind von $\mathrm{H}$. Altenmüller ausführlich beschrieben worden. ${ }^{63}$ Die Mumienriemen ("Lederbänder") sind der Mumie über Kreuz auf die Brust gelegt, ${ }^{64}$ und in ihre breiteren Enden ist ein Stück Pergament gesteckt, das ein mit einem Stempel eingeprägtes Bild- und Schriftfeld hat. Die Mumienanhänger bzw. "pendeloques”, die um den Hals der Mumie gehängt werden, bestehen aus einer Troddel, die mit einem Menat-Gegengewicht verbunden ist. Dieses Gegengewicht enthält oft eine eingeprägte Inschrift. Mumienriemen und -anhänger stammen eigentlich aus dem Götterkult, waren also zum Schmuck der Kultstatuen bestimmt, und sind erst sekundär auf die funeräre Sphäre übertragen worden. ${ }^{65}$ Die eingeprägten Inschriften bei den Mumienanhängern bestehen aus dem Namen des HPA mit Filiation, teilweise mit jrj.n davor. Die Mumienriemen haben vielfältigere Möglichkeiten: Darstellung des Hohepriesters anbetend vor dem Gott, dazu sein Name, oft mit Angabe des Vaters und teilweise mit jrj. $n$ davor; entsprechend der König (mit Kartusche) vor einem Gott, entweder allein oder mit Zusatz darunter: (jrj.n) HPA, z.T. mit Filiation. Mumienriemen und -anhänger sind nicht individuell für jedes Begräbnis gefertigt, sondern institutionell (also vom Tempel) hergestellt und ausgegeben worden. Zu ihrer Prägung muss es besondere Stempel für jede ausgebende Institution und jede "Regierungszeit" gegeben haben.

Die (beschrifteten) Leichenbänder sind hieroglyphisch beschrieben, meist mit der Formel mnht jrj.n HPA (+ Filiation) + Jahr. In zwei Fällen steht ungewöhnlicherweise ein Königsname (des Amenemope) davor. ${ }^{66}$

Die verschiedenen Textilien, Lederapplikationen und Amulette, mit denen eine Mumie ausgestattet ist, müssen nicht zur selben Zeit für diese Mumie angefertigt worden sein, sie können nachweislich aus verschiedenen Jahren stammen, die mehr als ein Jahrzehnt auseinanderliegen. Die Leichenbänder auf der Mumie des Hnzw-m3飞-hrw in Hamburg stammen aus den Jahren I I, I 2 und 23 Osorkons I., ${ }^{67}$ die auf der Mumie des Jmn-m-h3t aus der 2. Cachette ${ }^{68}$ u.a. aus dem Jahr $\mathrm{x}+3\left(5^{-8}\right)$ des Amenemope und dem Jahr 8 des Siamun. Es ist daher nicht erstaunlich, wenn eine Mumie Leichenbänder, Mumienriemen und -anhänger hat, die von verschiedenen Herrschern stammen, z.B. Leichenbänder des HPA Mencheperre und Mumienriemen Pajnedjems II., ${ }^{69}$ oder Mumienriemen von Amenemope und Pajnedjem II. und andere von Mencheperre. ${ }^{70}$ Es ist nicht ganz klar, wie diese verschiedenen Daten zu erklären sind. Vielleicht haben

\footnotetext{
62 Bestattungen A.43 und A.I43 in der 2. Cachette, s. Daressy, $A S A E$ 8, 25; 36-7; G. Maspero, Les momies royales de Déir el-Baharî (MMAF I.4; Paris, I 889 ), 565 .

$6_{3} \quad$ H. Altenmüller, 'Lederbänder und Lederanhänger von der Mumie des Chonsu-maacheru' in W. Köpke und B. Schmelz (eds), Alt-Ägypten (Mitteilungen aus dem Museum für Völkerkunde Hamburg, Neue Folge, Band 30; Hamburg, 2000), 73-112.

${ }_{64}$ Vgl. etwa Niwiński, Coffins, pl. XI, links; pl. XIV.

65 Altenmüller, in Köpke und Schmelz (eds), Alt-Ägypten, 82-4.

66 Daressy, $A S A E$ 8, 24 (A.38); 33 (A.124).

67 Altenmüller, in Köpke und Schmelz (eds), Alt-Ägypten, I I 7-23.

68 Bestattung A.r 24, s. Daressy, $A S A E$ 8, 33.

69 Bestattung A.98, s. Daressy, $A S A E$ 8, 30.

70 Bestattung A I I3, s. Daressy, $A S A E$ 8, 3 I
} 
die Tempel zum Zeitpunkt der Bestattung Ausrüstungsgegenstände unterschiedlichen Alters aus ihren Magazinen ausgegeben, oder man hat für die eigene Bestattung in verschiedenen Jahren Sachen in Auftrag gegeben. ${ }^{71}$

Die Herrscher auf diesen Mumienumhüllungen sind zunächst immer nur die oberägyptischen Regenten: Unter den Hohenpriestern Herihor, Pajnedjem I., Masaharta und Mencheperre (jedenfalls bis auf seine spätesten Jahre) sind auf thebanischen Mumien nur ihre Namen zu finden, nicht aber die der unterägyptischen Könige. Das ändert sich mit dem Beginn der Regierung des Amenemope: Von nun an sind auch die tanitischen Könige in Theben und Oberägypten bezeugt, wenn auch nicht durch eigene Denkmäler. ${ }^{72}$

Amenemope ist aber nicht nur der erste unterägyptische König, der wieder in Theben belegt ist, er ist offenbar eng mit Pajnedjem verbunden: Auf Mumien der 2. Cachette ist Amenemope zweimal allein bezeugt, ${ }^{73}$ einmal mit Smendes II., ${ }^{74}$ mit Mencheperre ${ }^{75}$ und Siamun, aber zehnmal zusammen mit Pajnedjem II., ${ }^{76}$ dazu zweimal ebenfalls mit Pajnedjem II. auf Mumienriemen aus anderen Bestattungen. ${ }^{77}$ Wo Amenemope mit Smendes, Mencheperre und Siamun auf den Hüllen ein und derselben Mumie genannt wird, sind das jeweils separate Stücke (z.B. Leichenband und Mumienriemen) mit eigenen Aufschriften, die nicht notwendig aus demselben Jahr stammen müssen. Mit Pajnedjem II. zusammen wird Amenemope dagegen zehnmal in ein und demselben Text genannt, dreimal auf Stoffbändern ${ }^{78}$ und siebenmal auf Mumienriemen. ${ }^{79}$ Das heißt, für die "Mumienriemen" gab es einen einzigen "Stempel” der Tempelverwaltung, auf dem beide Namen standen, und auch auf Leichenbändern erscheinen sie zusammen auf einem Exemplar.

Ein derartiges "Paar" aus König und Hohepriester ist sonst in der 2I. Dynastie unbekannt. Es sieht aus, als wären die beiden besonders eng verbunden gewesen, und das Mittel dazu könnte eben eine Heiratsallianz gewesen sein, indem der neue Hohepriester die Tochter des neuen Königs heiratete. Es dürfte mit dieser besonderen Beziehung zum König Amenemope zusammenhängen, dass Pajnedjem II. noch in anderer Hinsicht eine besondere Stellung unter den Hohepriestern der 2I. Dynastie hat: Er ist (abgesehen von Pianch) der einzige, der trotz seiner relativ langen Amtszeit (über 20 Jahre) keinerlei königliche Ambitionen ausdrückt, weder in Titeln und Epitheta noch in Bauten oder Restaurationen etc.

$71 \quad$ Vgl. Altenmüller, in Köpke und Schmelz (eds), Alt-Ägypten, i 19.

72 Amenemope ist bezeugt durch die oben behandelte Sphinxstatuette, die seine Tochter gestiftet hat, sowie in (privaten) Bestattungen (s.u.), Osochor durch eine Inschrift der "Priesterannalen" (Jansen-Winkeln, Inschriften, I, I I I [8.I]), Siamun durch Priesterannalen (Jansen-Winkeln, Inschriften, I, I 56 [9.57-8]), Wiederbestattungsvermerke (Jansen-Winkeln, Inschriften, I, I I4-I 7 [9. I 5; I9]), private Bestattungen (JansenWinkeln, Inschriften, I, I49-50 [9.42]; I 57 [9.59]) und ein Graffito in Abydos (Jansen-Winkeln, Inschriften, I, I 56 [9.56]), Psusennes II. durch Priesterannalen (Jansen-Winkeln, Inschriften, I, r62 [1 o. I0-1 I]; F. Payraudeau, 'De nouvelles annales sacerdotales de Siamon, Psousennès II et Osorkon Ier', BIFAO 108 [2008], 294; 307-8), ein Graffito (Jansen-Winkeln, Inschriften, I, I 58 [10.3]) und Scherben in Abydos (U. und A. Effland, Abydos, Tor zur ägyptischen Unterwelt [Mainz, 2013], 60-5).

73 Jansen-Winkeln, Inschriften, I, 107-8 (7.42; 7.47); bei Nr. 7.42 ist es möglich und bei $\mathrm{Nr} .7 .47$ sehr wahrscheinlich, dass der Kontext nicht erhalten ist, also der Königsname in Wirklichkeit mit einem anderen Herrschernamen vergesellschaftet war.

$74 \quad$ Bestattung A. 135, Daressy, ASAE 8, 35-6; Jansen-Winkeln, Inschriften, I, 107-8 (7.45).

75 Bestattungen A.ro5 [indirekt] und A. I 13, s. Daressy, $A S A E$ 8, 30-1.

76 Daressy, ASAE 8, 23-36; Jansen-Winkeln, Inschriften, I, 103-8 (7.34-9; 7.41, 43-44; 46).

77 Jansen-Winkeln, Inschriften, I, I09 (7.48-9).

78 Jansen-Winkeln, Inschriften, I, 103-4 (7.35; 7.37); 107 (7.44)

79 Jansen-Winkeln, Inschriften, I, Iо3-8 (7.34; 36; 39; 4I; 46; 48-9). 
Das Verhältnis von unter- und oberägyptischen Herrschern scheint sich also mit dem Regierungsantritt des Amenemope grundlegend gewandelt zu haben: Der König wurde nun in Theben als Oberherr anerkannt und der oberägyptische Regent beanspruchte keinen königlichen Rang mehr.

\section{v. Die oberägyptischen Herrscher in der 21. Dynastie}

Um diese Neuorientierung richtig zu verstehen, ist es nötig, sich einmal näher anzusehen, wo und wie die oberägyptischen Regenten bezeugt sind.

Vor der Zeit von Amenemope und Pajndejem II. gehen alle Aktivitäten, die man von einem ägyptischen König erwarten sollte, in Oberägypten nur von den eigenen Regenten aus, also von denjenigen Herrschern, die in Personalunion Militärbefehlshaber und Hohepriester sind. Das sind im einzelnen Herihor, Pajnedjem I., Masaharta ${ }^{80}$ und Mencheperre, die alle, in unterschiedlichem Ausmaß, königliche Titel und Attribute führen. In allen regulären Tempeldekorationen, bei der Stiftung von Statuen und Großplastik, in den Wiederbestattungsver-merken, bei der Austeilung der im Tempel hergestellten Ausrüstungen von Bestattungen (wie den Leichentüchern und -bändern, Mumienriemen etc.) werden grundsätzlich nur diese ober-ägyptischen Regenten erwähnt, nie die unterägyptischen Könige.

Man kann dagegen einwenden, wie es Kitchen getan hat, ${ }^{8 \mathrm{I}}$ dass es ja zumindest einen klaren Beleg für einen unterägyptischen König in Oberägypten gebe, nämlich die Felsstele des Smendes bei Dababieh (nahe Gebelein). Dies ist in der Tat eine formelle Felsinschrift, die in Form einer "Königsnovelle" berichtet, dass Smendes [Baumeister] und 3000 Arbeiter zu Hilfe schickte, als durch baulichen Verfall thebanische Tempel von Überflutung bedroht waren. Das könnte folgenden Hintergrund haben: Die Verlagerung der nördlichen Residenzstadt von Piramesse nach Tanis, das einzige Bauvorhaben der 21. Dynastie von großen Dimensionen, muss enorme Massen von Arbeitskräften erfordert haben, und für große Bauvorhaben in Ägypten wurden normalerweise Arbeiter aus dem ganzen Land herangezogen. ${ }^{82} \mathrm{Zu}$ dieser Zeit dürfte daher allein Smendes über Baumeister und Arbeiter in der nötigen Anzahl verfügt haben, und da das Verhältnis zwischen beiden Landeshälften freundschaftlich war, besteht kein Grund, warum er sie nicht zu Hilfe schicken sollte und warum seine Baumeister nicht eine Stele zu seinen Ehren anbringen konnten. Man kann daraus sicher nicht schließen, dass Smendes der "unangefochtene König von ganz Ägypten” war. $^{8}{ }_{3}$ In Theben wird Smendes nur einmal in einem nicht zur regulären Dekoration gehörigen kleinen Zusatz im Tempel des Month genannt. ${ }^{84}$ Wenn er wirklich als König für das ganze Land zuständig gewesen wäre, sollte man nach dem 'Tod Ramses' XI. ihn und nicht Herihor und Pajnedjem I. in der Dekoration des Chonstempels und der anderen thebanischen Tempeln erwarten. Aber Smendes erscheint nirgends in der

$80 \quad$ Noch zu Lebzeiten seines Vaters, also wohl als dessen Stellvertreter.

8I Kitchen, Third Intermediate Period, XVIII, § N.

82 M. Römer, 'Hauswirtschaft - Häuserwirtschaft - Gesamtwirtschaft: “Ökonomie” im pharaonischen Ägypten', Or 78 (2009), I6-I7; P. Andrassy, 'Zur Organisation und Finanzierung von Großbauten im Alten Ägypten', in M. Fitzenreiter (ed.), Das Heilige und die Ware (IBAES 7; London, 2007), I43-64.

${ }_{3}$ So Kitchen, Third Intermediate Period \$ 2 I4.

${ }_{4} \quad$ Jansen-Winkeln, Inschriften, I, I (1.2). Außerdem hat er in Abydos ein Votivgefäß stiften lassen, s. U. und A. Effland, Abydos, 58-9. Da Abydos für ganz Ägypten ein besonders heiliger Ort war, besagt das natürlich nichts über seinen Herrschaftsbereich. 
regulären Dekoration, und ebenso wenig Psusennes I., der eine sehr lange Regierung hatte, aber in Theben und Oberägypten überhaupt nicht belegt ist. ${ }^{8}$ Insgesamt ist es deutlich, dass in Oberägypten vor der Zeit des Amenemope in allen offiziellen Funktionen nur die oberägyptischen Regenten tätig wurden.

Das ändert sich mit der Regierung des Amenemope. Von nun an erscheinen die Regenten (Hohenpriester) nur noch auf Mumienausstattungen, z.T. neben dem König. Die „Priesterannalen“, die erst seit dieser Zeit belegt sind, nennen nur den König (im Datum). Neubauten und ,reguläre“ Tempeldekorationen gibt es in dieser Zeit überhaupt nicht mehr, entweder, weil die unterägyptischen Herrscher in Tanis daran kein besonderes Interesse hatten oder weil möglicherweise politisch unruhige Zeiten es nicht gestatteten. Wenn in Karnak in dieser Zeit noch längere Inschriften angebracht werden, ${ }^{86}$ sind es Orakeldekrete über Erbschaftsangelegenheiten und eine Art Orakelprozess mit anschließendem Freispruch, die eben nicht zur regulären Dekoration gehören und die auch nicht im Namen des Königs oder Hohenpriesters angebracht wurden. ${ }^{87}$

\section{Amenemope und Pajnedjem II.}

Es ist nicht unwahrscheinlich, dass die Verbindung zwischen Amenemope und Pajnedjem II. ${ }^{88}$ und die damit vermutlich einhergehende engere Anbindung Oberägyptens an das Königtum in Tanis die Reaktion auf irgendeine Krise war, sei es politischer oder dynastischer Art (oder beides). Es gibt immerhin einige Anzeichen, die für eine politische Krise und allgemein unruhige Zeiten sprechen: Der Hohepriester Smendes II. hatte nur eine ganz kurze Amtszeit gehabt, ${ }^{89}$ was immer ein verdächtiges Zeichen ist, möglicherweise ebenso der Vorgänger des Amenemope, falls Amenemnisut, wie es wahrscheinlich ist, der Nachfolger und nicht der Vorgänger Psusennes' I. war. Nach oder in diesem Jahr ändern sich die (Macht-)Verhältnisse offenbar. Es bleibt ohne jede konkrete Quelle dazu natürlich unbekannt, welcher Art eine solche Krise gewesen sein könnte: ob es etwas Dramatisches war, z.B. ein national-ägyptischer Aufstand in Oberägypten, oder eher Probleme innerhalb der Herrscherfamilie, etwa

85 Man könnte allerdings auf die Dachlastele (Z.I I) verweisen, wo in einem "Orakelprozess" eine Akte aus dem Jahr i 9 des "Pharao Psusennes” herangezogen wird, s. A. H. Gardiner, 'The Dakhleh Stela', $7 E A$ i 9 (1933), 22; pl. VI. Aber das wird seinen guten Grund haben: Die Grenze des thebanischen "Gottesstaates” bei El-Hibeh, zeigt, dass es bei der Aufteilung des Landes in der 2r. Dynastie nicht um die klassische Unterteilung in Ober- und Unterägypten ging, sondern dass den Königen in Tanis alle libysch besiedelten Gebiete unterstellt waren, und dazu zählten auch die Oasen.

86 Jansen-Winkeln, Inschriften, I, г 69-83 (г г.8-10).

87 Auch für die noch unpublizierte Stele aus Karnak mit einer Inschrift über ein Orakel für Nmrt A, p3 wr 3n $M$ Mr $n$ wrw p3j.k b3ksbq aus der Zeit des Siamun dürfte das zutreffen, s. G. Legrain, 'Rapport sur les travaux exécutés à Karnak', $A S A E_{5}$ (1904), 42-3; Payraudeau, BIFAO 108, 305; L. Coulon, 'Une stèle déposée par un grand chef Libyen près de la nécropole d'Osiris à Karnak', in C. Zivie-Coche und I. Guermeur (eds), 'Parcourir l'éternité': Hommages à fean Yoyotte (Turnhout, 201 2), $38 \mathrm{I}$.

88 Falls Amenemope der Sohn Psusennes' I. war (s.o., Anm. 38), waren die beiden Vettern.

$89 \quad$ Das berühmte Leichenband mit der Kartusche des Amenemope und einem Jahr 49 (G. Daressy, 'Contribution à l'étude de la XXIe dynastie égyptienne', Revue archéologique 28 [1896], 78; Jansen-Winkeln, Inschriften, I, I 08 [7.47]), das nur das Jahr 49 des Mencheperre sein kann (s.u., § 9), beweist, dass Amenemope am Ende der Amtszeit des Mencheperre König war. Da zwei Paare von dem Hohenpriester Smendes II. gestifteter Armbänder in der Bestattung Psusennes I. gefunden worden sind (Montet, Psousennès, I49, fig. 54; I 54-5 [Nr. 598-60I]; Jansen-Winkeln, Inschriften, I, 54 [4.50-I]), muss er noch zu Lebzeiten seines Vaters (ebenfalls) Hohepriester geworden sein, und er war damit zweifellos designierter Nachfolger als Regent von Oberägypten. Das hat in Anbetracht der langen Regierungszeit des Mencheperre, der sicher in hohem Alter stand, nichts Überraschendes. Auch Masaharta ist noch unter seinem Vater Hohepriester und Militärbefehlshaber geworden. 
mehrere Prätendenten, die sich bekämpften. Die oben vermutete enge Verbindung zwischen Amenemope und Pajnedjem II. würde zunächst eher auf ein dynastisches Problem deuten: Zwei jüngere Mitglieder der Herrscherfamilie(n) verbünden sich und setzen sich auf diese Weise möglicherweise gegen andere Prätendenten (oder die neuen Amtsinhaber) durch. Dafür könnte immerhin sprechen, dass sowohl Smendes II. wie auch Amenemnisut nach einer sehr kurzen Amtszeit verschwinden. Aber es ist wohlbekannt, dass gerade politisch (und militärisch) unruhige Zeiten häufig sehr kurze Regierungszeiten zur Folge haben. ${ }^{90}$ Man wird dann Machthaber, die sich als unfähig erwiesen hatten, schneller gestürzt haben, und allgemein dürfte man in solchen Zeiten weniger Hemmungen zu gewaltsamem Vorgehen gehabt haben. In einem thebanischen Papyrus aus einem Jahr 4 (des Amenemope oder eines seiner Nachfolger) ${ }^{91}$ wird ein vorhergehendes Jahr 49 als $h 3 w$ bjn, "schlechte Zeit" (bzw. "Hungersnot") bezeichnet. ${ }^{92}$ Es ist daher wohl wahrscheinlicher, dass es sich nicht (nur) um Streitigkeiten innerhalb der Herrscherfamilie gehandelt hat, sondern um ein umfassenderes Problem, dass sich vielleicht nur durch eine engere Zusammenarbeit zwischen den beiden Landesteilen und ihren Regenten lösen ließ.

Geht man von einer Absprache zwischen Amenemope und Pajnedjem II. aus, stellt sich auch die Frage, warum Pajnedjem bei dem eigenen Status offenbar zurückstecken musste bzw. wieso der oberägyptische Bereich nun seine Selbständigkeit verlor. Das könnte damit zu erklären sein, dass es eben ein oberägyptisches Problem war (z.B. ein Aufstand), das nur mit Hilfe des unterägyptischen Königs zu lösen war, dem man im Gegenzug Konzessionen machen musste. Vielleicht lag aber der "Heiratsallianz" (wenn es eine war!) auch eine umfassendere Absprache zugrunde: Der unterägyptische Familienzweig wurde zunächst der tonangebende, der Nachkomme aus der Verbindung von Pajnedjem und der Tochter des Amenemope wurde dafür Erbe beider Häuser. Auf diese Weise ließe sich die in der 2r. Dynastie so weit einzigartige Übernahme des Thrones in Tanis durch einen oberägyptischen Regenten erklären: allerdings nur, sofern der Hohepriester Psusennes ("III.”) und der gleichnamige letzte König der 21. Dynastie ("II.") dieselbe Person sind. Die Mutter Psusennes' III. ist allerdings nicht bekannt. Chronologisch wäre es immerhin möglich, dass er der Sohn der Tochter des Amenemope war, selbst wenn die Ehe erst nach der Thronbesteigung des Amenemope geschlossen wurde. Psusennes wäre dann etwa Mitte zwanzig gewesen, als er Hoherpriester wurde, ${ }^{93}$ und er wäre mit Mitte dreißig König geworden. ${ }^{94}$ Aber sind Psusennes II. und III. identisch?

\footnotetext{
$90 \quad$ Beispiele aus der ägyptischen Geschichte sind u.a. die spätere 20. Dynastie oder die Zweite Zwischenzeit. 91 R. Meffre, D'Héracléopolis à Hermopolis (Paris, 20I5), 257, n. 7I hat zurecht darauf hingewiesen, dass dieses Jahr 4 nicht notwendig der Zeit des Amenemope zuzuordnen ist, sondern durchaus später liegen könnte.

92 Papyrus Brooklyn r 6.205, 4,7, s. R. Parker, A Saite Oracle Papyrus from Thebes (BE Stud 4; Providence, 1962), 51, pl. I9; Jansen-Winkeln, Inschriften, I, I03 (7.33).

93 In der 22. Dynastie war der Hohepriester Osorkon B (der Sohn Takeloths II.) vermutlich noch jünger, als er sein Amt antrat, vgl. D. Aston, 'Takeloth II - a King of the "Theban Twenty-Third Dynasty"?', FEA 75 (1989), 150.

$94 \quad$ Falls es eine solche Absprache über die Thronfolge wirklich gegeben haben sollte, hätte man natürlichje nach Dauer der Regierungen von Osochor und Siamun—auch mit einer deutlich späteren Thronbesteigung rechnen müssen. Leider wissen wir nicht, wie Osochor und Siamun mit ihren Vorgängern und Nachfolgern verwandt waren.
} 


\section{König Psusennes II. und der Hohepriester Psusennes (“III.”).}

König Psusennes II. ist nach Manetho der letzte König der 2I. Dynastie, je nach Überlieferung mit I4 (Africanus) bzw. 35 (Eusebius) Jahren Regierungszeit. ${ }^{95}$ Eigene königliche Denkmäler hat er nicht hinterlassen. Er ist in Theben bezeugt durch Mumienriemen in den Begräbnissen A. 58 und A.66 der Bab el-Gasus, ${ }^{96}$ Datierungen der "Priesterannalen" 97 und eine Grabinschrift, ${ }^{8}$ in Abydos durch ein Graffito im Tempel Sethos' I. ${ }^{99}$ und Scherben von Votivgefäßen, ${ }^{100}$ in Memphis (vermutlich) durch eine Privatstatue, ${ }^{\text {Ior }}$ eine Perle und einige Einträge im pReinhardt $^{102}$ sowie postum durch Filiationen seiner Tochter $M 3^{\top} t-k 3-R^{\top}$ und seines Enkels Schoschenk. ${ }^{103}$ Er wurde vermutlich im Vestibül des Grabes Psusennes' I. (NRT III) (sekundär) bestattet, wie sich anhand der (sehr schlecht gearbeiteten) Uschebtis erschließen lässt, ${ }^{104}$ wenn auch kaum mit letzter Sicherheit. Als Schwiegervater Osorkons I. könnte er schon zur selben Generation wie Schoschenk I. gehört haben.

Für Psusennes II. ist neuerdings ein namentlich auf ihn bezogenes Regierungsjahr I I gesichert. ${ }^{105}$ Ein (anonymes) Jahr I $3_{3}$ könnte sich auf ihn, aber auch auf Siamun oder Schoschenk I. beziehen, ${ }^{106}$ ein weiterer Beleg für ein Jahr I3 könnte alternativ zu Schoschenk I. gehören ${ }^{107}$ und ein Jahr I4 ebenso zu Psusennes wie zu Siamun. ${ }^{108}$ Auch das Jahr i 9 auf der großen Dachlastele ${ }^{109}$ hat man Psusennes zurechnen wollen, ${ }^{\text {II0 }}$ aber die Gründe dafür sind kaum stichhaltig. ${ }^{\text {II }}$

Der Hohepriester Psusennes ("III.") ist als Sohn Pajnedjems II. der letzte bekannte Hohepriester der 21. Dynastie und wurde im Jahr io Siamuns Nachfolger seines Vaters. Er ist öfter auf Mumienriemen und -anhängern sowie Leichenbänder der Bab el-Gasus bezeugt. ${ }^{12}$ Neuerdings ist das Fragment eines Votivgefäßes aus

\footnotetext{
$95 \quad Z u$ den Schreibungen seiner Kartuschennamen (die anderen sind gar nicht belegt) s. U. und A. Effland, Abydos (Darmstadt, 2013), 63.

96 Jansen-Winkeln, Inschriften, I, I63 (го. 13-14).

97 Payraudeau, BIFAO I08, 293-308; Jansen-Winkeln, Inschriften, I, I62 (I0. I I).

98 Jansen-Winkeln, Inschriften, II, 37 ( I 2.5 I).

99 Jansen-Winkeln, Inschriften, I, I 58 (г 0.3 ); U. und A. Effland, Abydos, 6r.

100 U. und A. Effland, Abydos, 6I-3.

Iо $\mathrm{O}$ O. Perdu, Les statues privées de la fin de l'Egypte pharaonique (Paris, 2012), I98-207 (Nr. I 5).

102 Jansen-Winkeln, Inschriften, I, I 59 ( ( о.5); Jansen-Winkeln, Inschriften, I, I 96 ( I I.28); M. Römer, 'Die Entstehungszeit des Papyrus Reinhardt', GM 200 (2004), 83-4.

${ }_{103}$ Jansen-Winkeln, Inschriften, I, I82-3 (I I.IO); II, 57-8 (13.3 I-3). Auch der in der Ahnentafel Berlin 23673 zweimal erwähnte König Psusennes (Jansen-Winkeln, Inschriften, II, 278 [28.24]) könnte Psusennes II. sein, vgl. Kitchen, Third Intermediate Period, § I 53.

${ }_{104}$ J. Yoyotte, 'À propos de Psousennès II', BSFT I (I988), 4I-53; Tanis: L'or des pharaons, I36-7.

105 Payraudeau, BIFAO 108, 294; 307-8.

ro6 Maspero, Momies royales, 579; Jansen-Winkeln, Inschriften, I, I86 (I I.I 2); Kitchen, Third Intermediate Period, §391 (87).

${ }_{107}$ Fragment ${ }_{3} \mathrm{~B}$ der "Priesterannalen", s. G. Legrain, 'Notes prises à Karnak', RecTrav 22 (I900), 54; J.-M. Kruchten, Les annales des prêtres de Karnak (XXI-XXIIImes dynasties) et autres textes contemporains relatifs à l'initiation des prêtres d'Amon (OLA 32; Löwen, I989), 48; pl. 2; 17; Jansen-Winkeln, Inschriften, I, I62 (го.10); Kitchen, Third Intermediate Period, §391 (86).

108 Fragment 33 der "Priesterannalen", s. Legrain, RecTrav 22, 61; Kruchten, Les annales des prêtres de Karnak, I28; pl. I I ; 27; Jansen-Winkeln, Inschriften, I, I 56 (9.57); Kitchen, Third Intermediate Period, §390 (8I).

ro9 Gardiner, $\mathscr{F} E A$ i 9, 22; pl. VI.

rio R. Krauss, 'Das wrš-Datum aus Jahr 5 von Shoshenq [I]', DE 62 (2005), 43-8.

II Leahy, 'The Date of the "Larger" Dakhleh Stela (Oxford, Ashmolean Museum I894.107a)', GM 226 (2010), 50-1; Payraudeau, BIFAO г 08, 299-300.

${ }_{112}$ Bestattungen A.17; A.43; A.48; A.65; A.88; A.125; A.132-3; A.148, s. Daressy, ASAE 8, 23-37; Aston, Burial Assemblages, I 66-93; Jansen-Winkeln, Inschriften, I, I 50 (9.42); I62-3 (I0.1 2); I 90 (I I. I 7); 229-3 I (I I.8 I-6).
} 
Abydos bekannt gemacht worden, das sehr wahrscheinlich Psusennes III. zeigt. ${ }^{113}$ Mit dem Hohenpriester Psusennes sind zwei Daten assoziiert: ein Jahr I 2, das noch zur Regierungszeit des Siamun gehören könnte, ${ }^{114}$ und ein Jahr 5 , das sich nur auf den Nachfolger des Siamun beziehen kann. ${ }^{15}$ Allerdings ist die Lesung offenbar nicht ganz sicher. ${ }^{116}$

Es ist ein altes und öfter kontrovers diskutiertes Problem, ob der letzte König der 2 I . Dynastie und der Hohepriester, der auf Pajnedjem II. folgte, ein und dieselbe Person sind oder nicht. ${ }^{117}$

Gegen die Identität von König und HPA ist angeführt worden, dass nach dem Tod des Siamun und der Thronbesteigung Psusennes' II. in Theben ein neuer HPA zu erwarten sei, falls der alte König in Tanis geworden wäre. Ein solcher Nachfolger Psusennes' III. sei aber nicht bezeugt. ${ }^{\text {II }}$ Und falls das mit dem HPA Psusennes assoziierte Datum aus Jahr $5^{119}$ korrekt sei, wäre es schwer vorstellbar, dass man nach dem König Psusennes datiere, aber eben diesen König auf demselben Dokument als Hohenpriester bezeichne. ${ }^{\mathrm{I} 20}$ Es ist allerdings zu bedenken, dass es in der $2 \mathrm{I}$. Dynastie ja keinerlei parallelen Fall gab: Psusennes II. wäre der erste tanitische König, der zuvor als Regent von Oberägypten amtierte. Warum sollte er dann diese Funktion abgeben, wenn er König in Tanis wurde? Und in Theben konnte er eben weiterhin nach seiner alten Funktion benannt werden. ${ }^{\text {I2I }}$

Für die Identität des Königs und des HPA Psusennes spricht folgendes:

a) Die beiden letzten Regenten der oberägyptischen und der unterägyptischen Linie der 2I. Dynastie tragen denselben (eher seltenen) Namen.

b) Die Tochter $M 3^{\complement} t-k 3-R^{c}$ (B) des (tanitischen) Königs Psusennes hat Eigentum in Oberägypten, das von den Göttern Thebens garantiert wird. ${ }^{122}$

c) Der König Psusennes gründete ein $p r-M w t$ im ı。. oberägyptischen Gau in Ländereien unter der Verantwortung des thebanischen Amuntempels. ${ }^{123}$

d) Das bei weitem wichtigste Argument aber ergibt sich aus dem höchst ungewöhnlichen Graffito im Tempel von Abydos (10.3), ${ }^{124}$ in dem Königs- und HPA-Titel ineinander verschränkt sind (links königliche Titel, rechts Titel des Hohenpriesters und Militärführers):

${ }_{113}$ U. und A. Effland, Abydos, 64-5, Abb. II.

${ }_{14}$ Daressy, ASAE 8, 27 (65); Jansen-Winkeln, Inschriften, I, 230 ( I 1.83); Kitchen, Third Intermediate Period, $\S 390(80)$.

${ }_{115}$ Daressy, $A S A E$ 8, 23 (17); Jansen-Winkeln, Inschriften, I, I62-3 (10.12); Kitchen, Third Intermediate Period, §39I (85).

${ }_{116}$ In Revue archéologique 28, I 896, 77 ( ( 7) gibt Daressy "Jahr 4".

117 Ausführliche Darstellung der Belege und ihrer Interpretation bei J. Lull, 'Psusennes, primer sacerdote de Amón, y Psusennes II, rey de Egipto: genealogía, documentación y problemas', Aula orientalis 24 (2006), 57-77.

${ }_{11} 8$ Payraudeau, BIFAO i08, 303-4.

19 Daressy, ASAE 8, 23; Jansen-Winkeln, Inschriften, I, I62-3 (10.1 2); Kitchen, Third Intermediate Period, $\S 391(85)$.

120 Kitchen, Third Intermediate Period, $\$ 8$.

${ }_{121}$ In diesem Sinne jetzt auch Kitchen, Third Intermediate Period ${ }^{3}$, XXI-XXII ( $\$$ R-S).

122 Vgl. auch Kitchen, Third Intermediate Period, $\$ 238$.

123 Papyrus Reinhardt, V.30; VI.I I, s. S. Vleeming, Papyrus Reinhardt (Berlin, I993), 23; 24; JansenWinkeln, Inschriften, I, I 96 ( I I.28); vgl. Römer, GM 200, 83-4.

124 G. Daressy, 'Les temples d'Abydos', RecTrav 21 (1899), 9-1o; M. Murray, The Osireion at Abydos (BSAE 9; London, I 903), 36, pl. 2I; A. Dodson, 'Psusennes II', RdE 38 ( 1 987), 50-1; J. von Beckerath, Handbuch der ägyptischen Königsnamen (MÄS 49; Mainz, I999), I82-3; U. und A. Effland, Abydos, 6 r. 
Z.I:

nswt-bjt nb t3wj Tjt-hpr-R`Stp.n-Jmn-R` nswt ntrw

hm-ntr tpj $n$ Jmn-R` nswt ntrw

z3 R $n b h^{\ulcorner} w$

$h 3 w t j$

P3-sb3-hj-m-nwt Mrjj-Jmn

$n t j(r-) h 3 t n 3 m \check{s} w\left[{ }^{3} 3 w n K m t\right.$

$r-d r w]$

Z. 2

hm-ntr tpj $n$ Jmn-R ${ }^{\odot}$ nswt ntrw

jrj hpw nfrw $n$ Kmt ḩ3wtj

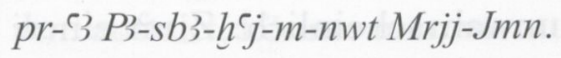

Eine ähnliche, wenn auch nicht so weitgehende, Verschränkung von Königsnamen und Titulatur des Hohenpriesters und Militärbefehlshabers findet sich bei Schoschenk (IIc), dem Enkel Psusennes' II. Auf der Nilstatue BM $8^{125}$ nennt er sich:

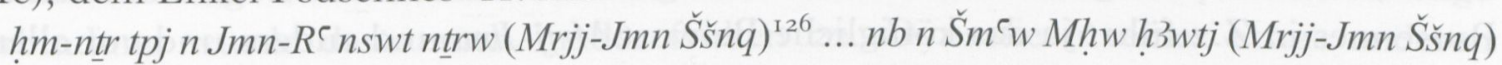

$n t j(r-) h 3 t n 3$ mšw $3 w n$ Kmt $r \underline{d r}$ rw z3 nswt nb t3wj nb jrj jht (Mrjj-Jmn Wsrkn).

Hier setzt der Hohepriester und Königssohn seinen Namen in Kartusche, mit dem königlichen Epitheton Mrjj-Jmn davor, und er nennt sich außerdem den "Herrn von Ober- und Unterägypten”. Im Graffito führt Psusennes II. in ZI zusätzlich die üblichen Königstitel nswt-bjt $n b t 3 w j z 3 R^{\top} n b h^{\complement} w$ sowie den Thronnamen. Es ist bemerkenswert, dass in beiden Fällen die Königstitulatur ausgerechnet mit demjenigen militärischen Titel vermischt wird, der in der 2I. Dynastie nur in kursiven Alltagstexten gebraucht wird, nie in monumentalen Inschriften. ${ }^{127}$

Es kann jedenfalls kein Zweifel daran bestehen, dass in Z.I des Graffitos Psusennes II. ${ }^{128}$ als (tatsächlicher) König und oberägyptischer Regent (Hoherpriester) genannt ist, während auf der Nilstatue sein Enkel als Regent mit königlichen Ambitionen (oder Vollmachten) ${ }^{129}$ auftritt, aber ohne eigentliche Königstitel.

F. Payraudeau, der das Graffito zuletzt besprochen hat, ${ }^{130}$ vermutet, dass es zwei Leute betrifft: in Z.I den König Psusennes, der vormals Hoherpriester war, also den Sohn Pajnedjems II., und in Z.2 seinen gleichnamigen Nachfolger als Hoherpriester (Abstammung unbekannt). Denn man müsse davon ausgehen, dass Psusennes (III.) sein Amt als Hoherpriester abgab, als er König wurde, so wie es auch Pajnedjem I.

I25 Jansen-Winkeln, Inschriften, II, 57-8 (13.32).

I26 Die runden Klammern stehen hier für die Kartusche.

${ }_{127}$ S. K. Jansen-Winkeln, 'Zum militärischen Befehlsbereich der Hohenpriester des Amun', GM 99 ( 987 ), 19, Nr. I-3 und 10 (wohl Smendes II., nicht III.). Erst ab der 22. Dynastie erscheint dieser spezifische Titel auch in Inschriften, nach der frühen 22. Dynastie ist er nicht mehr belegt und wohl außer Gebrauch gekommen.

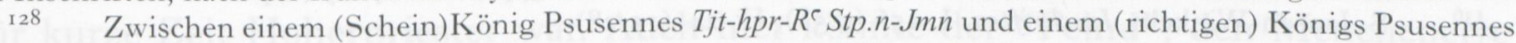
Tjt-hpr(w)-Re Stp. $n-R^{c}$ zu unterscheiden, wäre abenteuerlich, vgl. J. von Beckerath, 'Noch einmal Psusennes II.', $G M$ I30 (1992), I 7-I 9 .

${ }^{129}$ Man könnte aufgrund seiner Titel vermuten, dass er der militärische Oberbefehlshaber des ganzen Landes war.

130 Payraudeau, BIFAO 108, 303-4; ganz ähnlich A. Dodson, 'The Transition between the 21st and 22nd Dynasties Revisited', in G. P. F. Broekman, R. J. Demarée, and O. E. Kaper (eds), The Libyan Period in Egypt: Historical and Cultural Studies into the 2Ist-24th Dynasties. Proceedings of a Conference at Leiden University, 25-27 October 2007 (EU 23; Löwen, 2009), 106-7. 
und Masaharta gehalten hätten. Außerdem würden im Orakeldekret der Maatkare und im Papyrus Reinhardt Psusennes II. erwähnt und daneben ein (offenbar nicht mit ihm identischer) Hoherpriester. ${ }^{131}$ Das letztere ist allerdings ein Trugschluss: In dem Dekret werden in einer Aufzählung aller derjenigen, die es zu respektieren haben, als erste “jeder König” und “jeder Hohepriester des Amun” genannt. Daraus kann man nicht ernstlich folgern, dass zu dieser Zeit König und Hoherpriester unterschiedliche Personen waren, das Dekret soll ja für alle Zeiten gültig sein! Und im Papyrus Reinhardt wird einigemal ein König Psusennes erwähnt ${ }^{132}$ (sicher II.), und daneben die Institution des Hohenpriesters. ${ }^{133}$ Auch daraus kann man keinerlei Schlüsse über die jeweiligen Amtsinhaber ziehen.

Dennoch wäre es nicht mit letzter Sicherheit auszuschließen, dass es sich in Z.2 des Graffitos um einen zweiten Psusennes ("IV") handelt, der Psusennes III. (= II.) als oberägyptischer Regent gefolgt wäre, aber es ist sehr unwahrscheinlich: Zum einen behält ja Psusennes II./III. in Z. I den Titel des $h 3 w t j ~ . . . n t j ~ r-h 3 t$ ( ... ), der immer nur mit den oberägyptischen Regenten (und nie mit dem König) verbunden wird, zum anderen müsste ihm ein Regent nachgefolgt sein, der nicht nur denselben Namen führte, sondern auch gleich selbst wieder königliche Ambitionen hatte. Denn auch der Psusennes in Z.2 führt ja das königliche Beiwort Mrjj-Jmn und zudem und vor allem, was nicht einmal Schoschenk auf der Nilstatue tut, den Titel „Pharao“ ( $p r$ - 3 ) vor dem Namen! Zwar wollen Payraudeau ${ }^{134}$ und Dodson ${ }^{135}$ dieses pr-? mit h3wtj zu einem "Befehlshaber des Pharao" verbinden, aber die überaus häufig belegten Hohenpriester und Militärführer der 21. und 22. Dynastie nennen sich niemals h3wtj (n)pr-?3. Das pr-?3 ist eindeutig auf den folgenden Königsnamen bezogen, wie es auch sonst in der $2 \mathrm{I}$. und 22. Dynastie belegt ist. ${ }^{136}$ Auch Kitchen hat Z.2 des Graffitos zurecht so verstanden ("Pharaoh Psusennes (II) Meriamun"). ${ }^{137}$

Es werden also in beiden Zeilen des Graffito die Titel eines Königs und eines oberägyptischen Regenten namens Psusennes ineinander verschränkt genannt: In Z. I wird König Psusennes II. (Tjt-hpr-R`) eindeutig mit dem thebanischen Regenten (Hoherpriester und Oberbefehlshaber) identifiziert, umgekehrt in Z.2 der Regent ${ }^{13} 8$ mit dem Pharao. Es kann sich nur um ein und dieselbe Person handeln. ${ }^{\mathrm{I}}{ }^{9}$

Es bleibt natürlich die Frage, welchen Sinn diese eigenartige Mischung von Königsund Regententitulatur haben könnte. Ein Graffito ist keine “offizielle” oder reguläre Inschrift und stammt auch sicher nicht von einem Besuch des Königs selbst: Ein "Besuchergraffito", im Namen und mit Wissen des Königs angebracht, hätte man

${ }^{131}$ S. J. Lull, Los sumos sacerdotes de Amón tebanos de la whm mswt y dinastía XXI (ca. Io83-945 a.C.) (Oxford, 2006), 293.

${ }_{32}$ Vleeming, Papyrus Reinhardt, I 5 (II, I6); 23 (V,30); 24 (VI, I I); 44 (CC, I6).

133 Vleeming, Papyrus Reinhardt, i 8 (III, 24); 25 (VII, I9).

${ }^{1} 34$ Payraudeau, BIFAO io8, 303 ("le commandant de Pharaon").

${ }_{35}$ Broekman, Demarée und Kaper (eds), The Libyan Period in Egypt, 1 o6: "h3wtj (n) pr-?3".

${ }_{136}$ S. Jansen-Winkeln, Inschriften, I, I 56 (9.58); II, 24 (1 2.28).

137 Kitchen, Third Intermediate Period, XXI (§ R).

${ }_{13} 8$ Die Beiworte jrj hpw nfrw $n$ Kmt sind ganz ähnlich (allerdings auf Oberägypten beschränkt!) auch bei dem HPA Iuwelot aus der frühen 22. Dynastie belegt (Jansen-Winkeln, Inschriften, II, 80 [16.9], Z. I: $\underline{t z}$ hpw nfrw $\left.m t 3-\check{s} m^{\top} w\right)$.

I39 Die neuerdings veröffentlichte Scherbe eines Votivgefäßes aus Umm el-Qaab (U. und A. Effland, Abydos, 64-5, Abb. I I), auf der (sehr wahrscheinlich) der Hohepriester Psusennes III. zu sehen ist, hat für das Verständnis des Graffitos keinerlei Bedeutung, weil es aus der Zeit des Siamun stammen wird, als Psusennes eben nur Hoherpriester war. 
gewiss nicht über die alte Tempeldekoration geschrieben, und es handelt sich mit Sicherheit auch nicht um die "offizielle" Titulatur des Psusennes. ${ }^{140}$ Man könnte sich eher vorstellen, dass sich jemand über diese neue und völlig ungewohnte Kombination von oberägyptischem HPA und Militärbefehlshaber (in dessen Machtbereich Abydos lag) mit dem unterägyptischem König lustig machen wollte, indem man durch eine abstruse Mischung nicht zusammengehöriger Titel und Beiworte zunächst den König mit dem Regenten und dann den Regenten mit dem König gleichsetzte.

Psusennes II. führt öfter in der Kartusche vor dem Eigennamen bzw. zwischen MrijJmn und Eigennamen den Zusatz Hr. Es ist sicher, dass Psusennes (II.) und "HorPsusennes" ein und diesselbe Person sind, weil der Vater der Maatkare mit beiden Varianten genannt wird. Da Psusennes der einzige König dieser Zeit ist, der vorher thebanischer Hoherpriester war und andererseits dieser (königliche?) Zusatz nur bei ihm auftritt, könnte man vermuten, dass er etwas mit seiner "Beförderung” zum König zu tun hatte.

Wie auch immer, wenn der letzte oberägyptische Regent und der letzte unterägyptische König der 2I. Dynastie identisch waren, war damit eine noch engere Verklammerung beider Landesteile erreicht als unter Amenemope. Diese enge Verbindung wurde dann auch in der frühen 22. Dynastie in einer etwas anderen Weise fortgesetzt, indem jeweils ein Sohn des in Unterägypten regierenden Königs oberägyptischer Regent wurde, der somit nicht mehr einer eigenen "Dynastie” angehörte.

\section{viII. Theben und Oberägypten während der 21. Dynastie}

Eine "Geschichte" dieser Zeit, für welche Region auch immer, ist aufgrund mangelnder Quellen nicht zu schreiben (oder auch nur zu erkennen). Immerhin ist Theben noch derjenige Ort, aus dem die meisten Denkmäler dieser Zeit stammen und wo auch gewisse Aktivitäten bei der Dekoration von Tempeln zu erkennen sind. Dabei lassen sich, mit allem Vorbehalt, drei Phasen unterscheiden:

I. Unter Herihor und Pajnedjem I. (mit Masaharta) wird vergleichsweise (für Verhältnisse der 2 I. Dynastie!) viel unternommen: Unter Herihor sind große Teile des Chonstempels dekoriert und kleinere Arbeiten im Amuntempel geleistet worden, ${ }^{\text {III }}$ unter Pajnedjem I. wurde die Dekoration des Chonstempels fortgesetzt, (usurpierte) Großplastik im Amuntempel errichtet und verschiedene kleinere Vorhaben in anderen thebanischen Tempeln unternommen. In Koptos wurde eine große Steinstele usurpiert und neu beschriftet u.a.m. ${ }^{142}$ In dieser Phase sind nur diese Regenten, nicht die Könige in Tanis, in Oberägypten als Bauherren tätig. ${ }^{143}$

2. Die Stele der Verbannten deutet an, dass es am Ende der Regierung Pajnedjems I. Unruhen gab, die Mencheperre niederwarf, bevor er selbst vom Orakel als Hoherpriester eingesetzt wurde. Einige Zeit danach werden Verbannte begnadigt. Es ist nicht unwahrscheinlich, dass vor Mencheperre sein Bruder Djedchonsefanch für kurze Zeit Hoherpriester war. Auch hier könnte der "Feind”, den Mencheperre "vertrieb”, bevor er eingesetzt wurde, ein innerdynastischer Rivale sein (sein Bruder?)

\footnotetext{
140 Kitchens Vermutung (Third Intermediate Period, XXI, § R), das Graffito sei anlässlich eines Besuchs des Königs in Abydos angebracht worden, verkennt die Art dieses Textes und seiner Lokalisierung.

${ }^{141} \quad$ Vgl. die Zusammenstellung bei Römer, Gottes- und Priesterherrschaft, 35 .

142 Römer, Gottes- und Priesterherrschaft, $5 \mathrm{I}-4$.

${ }^{143}$ Zur Felsstele des Smendes s.o., $\S 5$.
} 
oder ein “äußerer” Feind, z.B. der Anführer eines Aufstandes. In der langen Amtszeit des Mencheperre hat es sehr wenig bauliche Aktivitäten in den thebanischen Tempeln gegeben; dafür sind mehrere Festungen gebaut oder ausgebaut worden. ${ }^{144}$ Das spricht eher dafür, dass es in Oberägypten selbst Schwierigkeiten gab, vermutlich Aufstände. Nicht ausgeschlossen, dass Mencheperres kurzfristiger Vorgänger dabei getötet wurde. ${ }^{145}$ Die zweite Phase, unter dem Regenten Mencheperre, scheint also, zumindest in Oberägypten, von zunehmenden Schwierigkeiten geprägt zu sein. Auch in dieser Phase gehen aber alle herrscherlichen Aktivitäten, die wir erkennen können, vom oberägyptischen Regenten aus. Die Könige von Tanis sind in Oberägypten in dieser Zeit nicht bezeugt.

3. Die dritte Phase beginnt mit Amenemope und ist zunächst durch die oben geschilderten strukturellen Änderungen gekennzeichnet, die vermutlich die Antwort auf eine kritische politische Situation waren (s.o., § 6). In dieser Phase ist so gut wie keine bauliche Tätigkeit mehr festzustellen. Das erste und einzige Denkmal in Karnak nach Mencheperre bleibt der Schriftzug der Tochter des Amenemope auf der Sphinxstatuette. Da nun aber die unterägyptischen Könige die offiziellen Landesherren sind, bleibt ungewiss, ob dies auf deren Desinteresse zurückzuführen ist oder ob die Verhältnisse auch nach der Intervention(?) des Amenemope unsicher geblieben sind. Falls die I 53 Bestattungen der 2. Cachette (Bab el-Gasus), wie Niwiński vermutet hat, ${ }^{146}$ nicht sukzessive dort beigesetzt worden sind, sondern zusammen in einer einzigen Aktion, könnte das wiederum ein Indiz für unsichere Verhältnisse sein.

Insgesamt dürfte eine solche Skizze der herrscherlichen Aktivitäten zumindest erahnen lassen, dass die politischen Verhältnisse während der 21. Dynastie recht labil waren.

\section{Ix. Die Datierungen der 21. Dynastie}

Die Datierungen der 2r. Dynastie ${ }^{147}$ sind in mancherlei Hinsicht bemerkenswert, und bestimmte Eigenheiten werden nicht auf den ersten Blick deutlich. Es ist daher nötig, sie sich einmal insgesamt anzusehen.

Zunächst handelt es sich ganz überwiegend um Datierungen aus Theben, mit nur drei Ausnahmen: das sind eine Stele aus Memphis mit einer Urkunde aus

\footnotetext{
144 In El-Hibeh (A. J. Spencer, Brick Architecture in Ancient Egypt [Warminster, I 979], I45; pls 34-5, nos 85-7; 89; Jansen-Winkeln, Inschriften, I, 80 [6.18]); Nazlat esch-Schurafa (M. Chabân, 'Fouilles à Achmounéîn', ASAE 8 [1907], 223 [IV]; G. A Wainwright, 'El Hibah and Esh Shurafa and Their Connection with Herakleopolis and Cusae', ASAE 27 [1927], 76-7; Spencer, Brick Architecture, I45, pl. 34, no. 82; Jansen-Winkeln, Inschriften, I, 80 [6.19]); Higazeh (Spencer, Brick Architecture, pl. 35, no. 92; Jansen-Winkeln, Inschriften, I, 80 [6.20]) und Gebelein (Spencer, Brick Architecture, ro9, I45, pl. 34, nos 83-4, pl. 35, no. 88; Jansen-Winkeln, Inschriften, I, 8 I [6.22]). In Karnak-Ost sind die Reste einer aus Ziegel (mit dem Namen des Mencheperre) gebauten "Bastion" gefunden worden, die wohl Teil einer befestigten Umfassungsmauer war (Karnak X, I995, 213-5). In Theben und Umgebung sind eine ganze Reihe von Ziegeln gefunden worden, die mit dem Namen des Mencheperre oder seiner Frau 3st-m-3hbjt gestempelt waren, z.B. in Karnak (Jansen-Winkeln, Inschriften, I, 78-9 [6.8]), Luxor (Spencer, Brick Architecture, 145, pl. 34, no. 83, pl. 35, no. 9I; Jansen-Winkeln, Inschriften, I, 79 [6.10] und Medamud (Spencer, Brick Architecture, I45, pl. 34, no. 82, pl. 35, no. 90; Jansen-Winkeln, Inschriften, I, 8 I [6.2 I]). Es ist wohl anzunehmen, dass sie aus ähnlichen festungsartigen Umfassungsmauern der Tempel stammen.

${ }^{145}$ J. Von Beckerath, 'Die "Stele der Verbannten”" im Museum des Louvre', $R d E_{20}$ ( 1968 ), 32, n.3 vermutet, er könnte von einem Usurpator getötet worden sein.

ז46 'The Bab El-Gusus Tomb and the Royal Cache in Deir el-Bahri', $\mathscr{F E A} 70$ (1984), 76.

${ }^{147}$ S. Kitchen, Third Intermediate Period, \$ 379-39 I; Jansen-Winkeln, Inschriften, I, 286-8.
} 
dem Jahr r 6 des Siamun, ${ }^{148}$ ein Felsgraffito aus Abydos aus dem Jahr i 7 desselben Herrschers ${ }^{149}$ und eine Wiederbestattung aus Kom Ombo. ${ }^{150}$

Die thebanischen Datierungen sind zumeist anonym, also nicht namentlich auf einen Herrscher bezogen; nur 13 unter ihnen (von mehr als 80) nennen ihn. Das sind erstens die seit Osochor bezeugten Priestereinführungsinschriften von Karnak ("Priesterannalen": sieben Belege in der 21. Dynastie), ${ }^{\mathrm{I}}{ }^{\mathrm{I}}$ dann aus der Zeit des Siamun zwei Leichenbänder und ein Sarg “docket" ${ }^{52}$ sowie ein (etwas mysteriöses) "Dokument” aus der Sammlung Golenischeff, das aber jedenfalls zur Bestattung der Ns-Hnzw A gehören muss, ${ }^{153}$ ein Totenbuch aus Jahr 5 des Amenemope ${ }^{154}$ sowie ein Leichenband aus dem Jahr 48 des HPA Mencheperre. ${ }^{155}$

Dieses letztere Leichenband ist der früheste Beleg für eine explizite Datierung in der 2 I. Dynastie überhaupt. Auf der Mumie, von der dieses Band stammt (Bestattung A.ro5 des Wsr-h3t-msw aus der Bab el-Gasus), war auch ein weiteres, das nur das Datum Jahr I, 4. 3ht, Tag I trug, ${ }^{156}$ und dieses Datum kann sich nach Lage der Dinge wohl nur auf den König Amenemope beziehen. Es ist sehr bemerkenswert, dass es explizite Datierungen erst genau seit der Zeit gibt, als der oben $\left(\S_{4}-6\right)$ beschriebene Wandel in Theben eintrat. Es liegt nahe, hier einen Zusammenhang zu vermuten. Da vor diesem Zeitpunkt nur die oberägyptischen Regenten in Theben (und zweifellos in Oberägypten überhaupt) offiziell aktiv waren (s.o.) und diese Regenten bis zu eben diesem Zeitpunkt auch alle gewisse "königliche" Attribute führten, liegt die Vermutung nahe, dass sich die Datierungen vor Amenemope auf die oberägyptischen Regenten beziehen. Das würde zum einen erklären, dass es vorher nur anonyme Datierungen gab, weil der Bezug eben zweifelsfrei war, und zum anderen, dass man genau ab dem Zeitpunkt explizite Datierungen findet, als dieser Bezug sich änderte bzw. nicht mehr selbstverständlich war.

Ausgehend von einer Reversion der bislang angenommenen Reihenfolge der Hohepriester Herihor und Pianch, hatte ich schon 1992 einen entsprechenden Vorschlag gemacht (der besser zu der neuen Abfolge Pianch - Herihor passt), nämlich die mit

${ }_{148}$ Kairo JE 70218, s. H. Munier, 'Un achat de terrains au temps du roi Si-Amon', in Recueil d'études égyptologiques dédiées à la mémoire de fean-Francois Champollion (Paris, 1922), 36I-6; Jansen-Winkeln, Inschriften, I, I 50-1 (9.43); Kitchen, Third Intermediate Period $\$ 390$ (82). Eine verbesserte Abschrift und Übersetzung wird C. Jurman demnächst vorlegen.

${ }_{449}$ H. Gauthier, Le livre des rois d'Egypte, III (MIFAO I9, I913) 295; Jansen-Winkeln, Inschriften, I, I 56 (9.56); Kitchen, Third Intermediate Period \$ 390 (84); U. und A. Effland, Abydos, 59-60, Abb. 5.

${ }_{150}$ S. Wenig, 'Eine Grabkammer des Mittleren Reiches aus Kom Ombo', FuB io (i 968 ), 87; K. JansenWinkeln, 'Zu einer Sekundärbestattung der 2I. Dynastie in Kom Ombo', GM 202 (2004), 7I-8; Jansen-Winkeln, Inschriften, I, $94(6.45)$.

${ }_{151}$ Jahr 2 Osochor: Legrain, RecTrav 22, I 900, 53 (3B); Kruchten, Les annales des prêtres de Karnak, 45-7, pl. 2, I 7; Kitchen, Third Intermediate Period, § 388 (56); Jahr I4 Siamun: Legrain, RecTrav 22, I900, 6I (33); Kruchten, Les annales des prêtres de Karnak, I28, pl. I1, 27; Kitchen, Third Intermediate Period, §390 (8I); Jahr I 7 Siamun: Legrain, RecTrav 22, 53-4 (3B); Kruchten, Les annales des prêtres de Karnak, 47-8, pl. 2, I7; Kitchen, Third Intermediate Period, $\$ 390$ (83); Jahr [X] Siamun: Payraudeau, BIFAO I 88, 294, 307-8; Jahr I I Psusennes II.: Payraudeau, BIFAO Io8; Jahr ${ }_{3} 3$ [Psusennes II.?]: Legrain, RecTrav 22, 54 (Z.6); Kruchten, Les annales des prêtres de Karnak, 48, pl. 2, I7; Kitchen, Third Intermediate Period, § 390 (86); [Jahr X] Psusennes II.: Legrain, RecTrav 22, 58 (1 7); Kruchten, Les annales des prêtres de Karnak, 103-4, pl. 7, 24.

${ }_{152}$ Jansen-Winkeln, Inschriften, I, I 50 (9.42); I 57 (9.59); I I4-I 7 (9.1 5): Kitchen, Third Intermediate Period, $\S 389(73 ; 74 ; 77)$.

${ }_{153}$ O. Berlev, 'The Date of pPrakhov', GM I60 (1997), 6.

${ }^{154}$ S.o., Anm. 56.

155 Daressy, Revue archéologique 28, 77; Daressy, ASAE 8, 30; Jansen-Winkeln, Inschriften, I, 90 (6.38),

${ }_{156}$ Daressy, ASAE 8, 30; Jansen-Winkeln, Inschriften, I, Io6 (7.40); Kitchen, Third Intermediate Period, $\S 388(5 \mathrm{I})$. 
Herihor, Pajnedjem I. und Mencheperre zeitgleichen Datierungen auf sie selbst zu beziehen und nicht auf die tanitischen Könige. ${ }^{157}$ Dieser Vorschlag ist weitestgehend auf Ablehnung gestoßen, auch bei denjenigen, die diese neue Reihenfolge Pianch Herihor akzeptiert hatten. Ich möchte daher noch einmal das pro und contra kurz resümieren und auf die Einwände eingehen:

a) Wie oben gesehen, sind die oberägyptischen Regenten (Hohenpriester) Herihor, Pajnedjem I. und Mencheperre ausschließlich für alle "königlichen” Angelegenheiten in Oberägypten zuständig, nicht die unterägyptischen Könige. Es gibt keinen Grund, warum man nicht nach diesen (königlichen!) Regenten hätte datieren sollen, die alle entsprechenden Aufgaben in Oberägypten wahrnahmen und die alle auch königliche Titel oder Attribute führten. Und weil sie das tun, liegt Kitchens Einwand ${ }^{158}$ neben der Sache, in Ägypten habe man seit jeher, und auf alle Fälle von der I 2. Dynastie bis zu den Ptolemäern, immer nur nach Königen ("in reality or by claim”) datiert und nicht nach irgendwelchen "civic/religious non-royalties". Die oberägyptischen Regenten Herihor, Pajnedjem I. und Mencheperre sind nach Anspruch und Praxis eindeutig "royalties". ${ }^{59}$ Der Fehler liegt im Gegenteil darin, sie für bloße "Hohepriester" in der Tradition des Neuen Reiches zu halten, wie das in der älteren ägyptologischen Literatur üblich war.

b) Es gibt eine komplementäre Verteilung: In der ersten Hälfte der 21. Dynastie findet man in Oberägypten "königliche" Regenten, anonyme Datierungen und keine unterägyptischen Könige, ${ }^{160}$ in der zweiten Hälfte sind unterägyptische Könige in Theben belegt, und auf sie werden auch die Datierungen (zum Teil namentlich) bezogen. Entsprechend führen die oberägyptischen Regenten keine königlichen Attribute mehr. ${ }^{161}$ Dort, wo es in den Quellen keine expliziten Aussagen gibt, ist eine komplementäre Distribution immer ein solider Hinweis auf einen strukturellen Unterschied. Die Abwesenheit unterägyptischer Könige genau in dem Zeitraum, wo die oberägyptischen Herrscher königliche Titel beanspruchen, wird doch kein Zufall sein.

c) Explizite, namentliche Datierungen gibt es genau ab dem Zeitpunkt, als sich Macht- oder Rechtsverhältnisse ändern und zum ersten Mal unterägyptische Könige in offizieller Funktion auftreten. Wäre schon immer nach ihnen datiert worden, hätte man es bei den anonymen Datierungen belassen können.

d) Zumindest eine explizite Datierung, ein Jahr 48, ist namentlich auf den Hohepriester Mencheperre bezogen. Kitchens Gegenvorschlag, ${ }^{162}$ man habe hier die Jahreszählung des Königs Psusennes auf Mencheperre bezogen (so wie Hatschepsut die Datierung nach Thutmosis III. übernommen habe), ist bestenfalls eines kühne Behauptung. Wenn Psusennes gemeint war, warum nennt man ihn nicht, und wenn

\footnotetext{
${ }_{157}$ Jansen-Winkeln, 'Das Ende des Neuen Reiches', $Z \ddot{A} S$ i 99 (1992), 34-7.

${ }^{15}$ Kitchen, Third Intermediate Period, XVII ( $\left.\$ \mathrm{~L}\right)$; ebenso in Broekman, Demarée und Kaper (eds), The Libyan Period in Egypt, 192.

${ }_{159}$ Da die "Amtszeit" des Masaharta unzweifelhaft und unstrittig (nach jedwedem Datierungssystem) in die Lebens- und Regierungszeit seines Vaters Pajnedjems I. fällt, versteht es sich von selbst, dass die zusammen mit seinem Namen auftretenden anonymen Datierungen auf Pajnedjem und nicht auf Masaharta zu beziehen sind; es stellt keinerlei "Ausnahme” dar (so Kitchen, Third Intermediate Period, XVIII [§ M]).

r60 Jedenfalls nicht in den üblichen königlichen Funktionen, z.B. als Bauherr und Ritualvollzieher im Tempel. Zur oberägyptischen Felsstele des Smendes, die Kitchen (Third Intermediate Period, XVIII [§ N]) als Beweis dafür anführt, dass Smendes auch der "Landesherr" von Oberägypten war, s.o., § 5.

I61 Vgl. K. Jansen-Winkeln, 'Die thebanischen Gründer der 2r. Dynastie', GM I 57 ( 1997 ), 69-72 (I 2).

${ }_{162}$ Kitchen, Third Intermediate Period, XVII-XVIII ( $\left.\$ \mathrm{M}\right)$; Kitchen, in Broekman, Demarée und Kaper (eds), The Libyan Period in Egypt, 192.
} 
man schon immer nach den unterägyptischen Königen datiert hatte, wieso wird dann hier sein Name ersetzt? Es ist richtig, dass es für eine namentlich auf einen Regenten bezogene Datierung nur diesen einen Beleg gibt. Aber in dieser gesamten Zeit, als es königliche Regenten in Oberägypten gab (Herihor bis Mencheperre), gibt es keine einzige Datierung, die auf einen unterägyptischen König bezogen ist!

e) Ein gewichtigerer Einwand scheint die zusammen mit Pajnedjem I. belegte Kette von Daten zu sein:

Als Hoherpriester wird Pajnedjem in den Belegen aus den Jahren $6,{ }^{163} 7,{ }^{164} 9,{ }^{165}$ Io $(2 \mathrm{x}),{ }^{166} \mathrm{I} \mathrm{I}^{167}{ }^{12} 2,{ }^{168}{ }^{1} 3^{169}$ und ${ }^{1} 5^{170}$ bezeichnet. In diese Reihe gehören auf jeden Fall auch die zusammen mit dem HPA Masaharta genannten Datierungen aus den Jahren $16,{ }^{171}{ }_{1} 8^{172}$ und $19,{ }^{173}$ da Masaharta unstrittig zu Lebzeiten seines Vaters im Amt war und im Jahr 25 schon Mencheperre als Hoherpriester eingeführt wurde. Als König wird Pajnedjem I. in zwei Datierungen aus einem Jahr 8 erwähnt. ${ }^{174}$ Kitchen bezieht die Daten mit den Hohenpriestern Pajnedjem und Masaharta auf Smendes I., das Jahr 8 mit dem König Pajnedjem aber auf Psusennes I. Er schließt aus ihrer Reihenfolge, dass Pajnedjem bis zum Jahr I5 des Smendes Hoherpriester war, dann dieses Amt seinem Sohn Masaharta abtrat und selbst König wurde. Daher müsse sich das Jahr 8 mit König Pajnedjem auf einen Nachfolger des Smendes beziehen, als der nur Psusennes I. in Betracht kommt.

Wenn sich die Daten der ersten Hälfe der 2r. Dynastie aber auf die oberägyptischen Herrscher beziehen, kommt für diese Serie nur Pajnedjem I. selbst in Frage. Damit ergäbe sich die Schwierigkeit, dass die Daten der Jahre 6 und 9-1 5 ihn als Hohenpriester bezeichnen, die aus dem Jahr 8 aber als König. Dies ist aber nur ein scheinbares Problem, da es überaus deutlich ist, wie unterschiedslos gerade bei Pajnedjem I. königliche und nichtkönigliche Titel und Attribute benutzt werden, zumindest in seinen früheren Jahren. Es kann sein, dass er mit dem Amtsantritt des Masaharta als Hoherpriester selbst nur noch als König bezeichnet wurde; zumindest postum wird er immer nur als König erwähnt. Vorher sieht das aber ganz anders aus: ${ }^{175}$ Im Luxortempel wird

I63 Maspero, Momies royales, 536-7, fig. 8, 545-6, fig. I4; Jansen-Winkeln, Inschriften, I, 21 (3.29-30); Kitchen, Third Intermediate Period, $\$ 38 \mathrm{I}(9 ; \mathrm{r} 0)$.

${ }_{164}$ Maspero, Momies royales, 54I; Jansen-Winkeln, Inschriften, I, 2I (3.3 I); Kitchen, Third Intermediate Period, $\$ 386$ (39). Der Wiederbestattungsvermerk auf der Mumie der Prinzessin Z3t-k3-msjw aus einem Jahr 7 stammt mit größter Wahrscheinlichkeit aus derselben Datierungsserie.

r65 Maspero, Momies royales, 564; Jansen-Winkeln, Inschriften, I, 23 (3.37); Kitchen, Third Intermediate Period, $\S_{3} 8 \mathrm{I}$ (12).

${ }_{166}$ Maspero, Momies royales, 554-5; 564; Jansen-Winkeln, Inschriften, I, 23 (3.37, Anm.; 3.38); Kitchen, Third Intermediate Period, $\S 38$ I ( $13 ;$ I4).

${ }_{167}$ Jansen-Winkeln, Inschriften, I, $3_{8}$ (3.67); Kitchen, Third Intermediate Period, $\S_{3} 82$ ( I 7 ).

i68 V. Loret, 'Le tombeau d'Aménophis II', BIE III, 9 (I898), I09; Jansen-Winkeln, Inschriften, I, 22 (3.34); Third Intermediate Period, $\S 382$ (22).

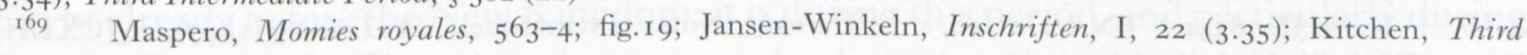
Intermediate Period, $\S_{382}(25)$.

${ }_{170}$ Maspero, Momies royales, 560; fig. 18; Jansen-Winkeln, Inschriften, I, 22-3 (3.36); Kitchen, Third Intermediate Period, $\S_{3} 82(26)$.

${ }_{171}$ Maspero, Momies royales, 536-7; fig. 8; Jansen-Winkeln, Inschriften, I, 28 (3.5I); Kitchen, Third Intermediate Period, $\S 383$ (27); Jansen-Winkeln, Inschriften, I, 39 (3.75); Kitchen, Third Intermediate Period, $\S$ $383(28)$.

${ }_{172}$ Jansen-Winkeln, Inschriften, I, 28-9 (3.53); Kitchen, Third Intermediate Period, $\S_{3} 8_{3}$ (29).

173 Jansen-Winkeln, Inschriften, I, $28(3 \cdot 52)$; Kitchen, Third Intermediate Period, $\S_{3} 83(30)$.

${ }_{174}$ Maspero, Momies royales, 534, fig. 7, 538, fig. 9; Jansen-Winkeln, Inschriften, I, 22 (3.32-3); Kitchen, Third Intermediate Period, $\S 386(40 ; 41)$.

${ }_{175}$ Vgl. dazu Jansen-Winkeln, GM I 57, 72-3 sowie Kitchen selbst, Third Intermediate Period, $\$ 215$. 
er in einer Szene als Hoherpriester bezeichnet, ${ }^{17^{6}}$ drei seiner Töchter aber als $z 3 t$ $n s w t ~ n \underline{h t}$.f. In Medinet Habu ist er General und Hoherpriester, seine Frau hingegen als Königin dargestellt und als $n b t$ t3wj bzeichnet, mit ihrem Namen in Kartusche. ${ }^{177}$ Auf der Statuette Kairo CG 42I9I ist er mit Uräus beim Weinopfer dargestellt, aber

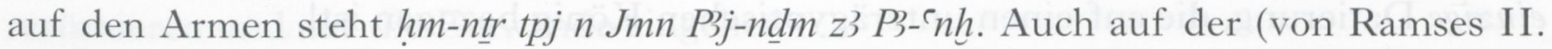
usurpierten) königlichen Kolossalstatue ${ }^{178}$ wird er so genannt. Im Chonstempel führt Pajnedjem den Horusnamen K3-nht Mrjj-Jmn, dahinter heißt er nswt-bjt shtp ntrw jrj 3hw

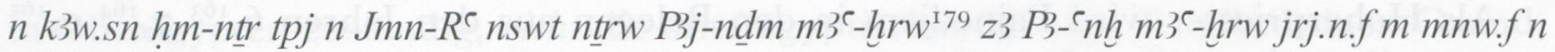
jtj.f Hnzw ... etc. ${ }^{180}$ Es ist also durchaus nicht überraschend, wenn er schon in seinem Jahr 8 einmal mit seiner königlichen Titulatur bezeichnet wird. ${ }^{181}$

Die Einordnung des Jahres 8 in die Reihe der übrigen Datierungen mit Pajnedjem und Masaharta wird auch durch folgende Überlegung nahegelegt: Datierte Vermerke ihrer Zeit auf wiederbestatteten Särgen oder Mumien von Königen des Neuen Reiches sind aus den Jahren $6(2 \mathrm{x}), 7,8(2 \mathrm{x}), 9$, Iо, I 2, I3, I 5, I6, I 8 und I 9 belegt. Es ist doch sehr wahrscheinlich, dass diese Jahre eine einzige Kette (von I3-I4 Jahren) bilden und auf eine einzige Regierungszeit bezogen sind, umso mehr, als es sich bei den Wiederbestattungen aus den Jahren 6, 7 und 8 (mit Pajnedem als König) jeweils um Mumien der frühen i 8. Dynastie handelt (Ahmose, Prinz Siamun, Prinzessin Ahmose-Satkamose, Amenophis I., Thutmosis II.), die vermutlich alle nicht aus dem Tal der Könige stammten. ${ }^{182}$ Nach Kitchens System folgte dagegen auf die Wiederbestattungen in den Jahren $6(-7)$ und 9-I 9 des Smendes eine Lücke von I 7-I 8 Jahren (die Jahre 20-26 Smendes' I., I-4 Amenemnisut, I-7 Psusennes' I.), dann zwei (oder drei $)^{183}$ Wiederbestattungen im Jahr (7 und) 8 Psusennes' I.

Insgesamt gibt es eine Reihe von Argumenten dafür, die oberägyptisch / thebanischen Daten von Herihor, Pajnedjem I. und Mencheperre auch auf diese Herrscher zu beziehen und nicht auf die Könige von Tanis, und umgekehrt sehe ich nichts, was ernsthaft dagegen spräche.

${ }_{176}$ Jansen-Winkeln, Inschriften, I, I7 (3.22a).

177 Kairo JE $3834 \mathrm{I}=$ TN 2/2/21/10, s. G. Daressy, 'Notes et remarques', Rec Trav 19 (1897), 20 (CXLII); Jansen-Winkeln, Inschriften, I, 20-I (3.27).

${ }_{178}$ L. Habachi, The Second Stela of Kamose (ADAIK 8; Mainz, I972), I6-20, pl. II; Jansen-Winkeln, Inschriften, I, 5-6 (3.4).

${ }_{179} M 3^{C}$-hrww kann in diesem Zusammenhang natürlich nicht bedeuten, dass Pajnedjem zu diesem Zeitpunkt verstorben war.

I80 Es gibt noch eine Reihe von weiteren Belegen für den königlichen Status des Hohenpriesters Pajnedjem.

I81 Vgl. auch: G. Demidoff, 'Hérihor-Piankhy, Piankhy-Hérihor: Retour sur une controverse', in: C.

Gallois, P. Grandet, und L. Pantalacci (eds), Mélanges offerts à François Neveu (BdE I45; Kairo, 2008 ), 106.

${ }_{182}$ Vgl. N. Reeves, Valley of the Kings (London, I990), 25I-4.

${ }_{1} 8_{3}$ In Kitchens Datierungssystem kann man die Wiederbestattung der Satkamose ebenso gut unter Smendes oder Psusennes ansetzen. 